\title{
Gradhiva
}

GRADHIV

Revue d'anthropologie et d'histoire des arts

$14 \mid 2011$

Carl Einstein et les primitivismes

\section{Le Cavalier bleu, 1931}

\section{Carl Einstein}

Traducteur : Isabelle Kalinowski

\section{CpenEdition}

\section{Journals}

Édition électronique

URL : http://journals.openedition.org/gradhiva/2206

DOI : 10.4000/gradhiva.2206

ISSN : 1760-849X

Éditeur

Musée du quai Branly Jacques Chirac

Édition imprimée

Date de publication : 30 novembre 2011

Pagination : 204-229

ISBN : 978-2-35744-046-3

ISSN : 0764-8928

\section{Référence électronique}

Carl Einstein, « Le Cavalier bleu, 1931 », Gradhiva [En ligne], 14 | 2011, mis en ligne le 30 mai 2012 consulté le 19 avril 2019. URL : http://journals.openedition.org/gradhiva/2206 ; DOI : 10.4000/ gradhiva.2206

(C) musée du quai Branly 


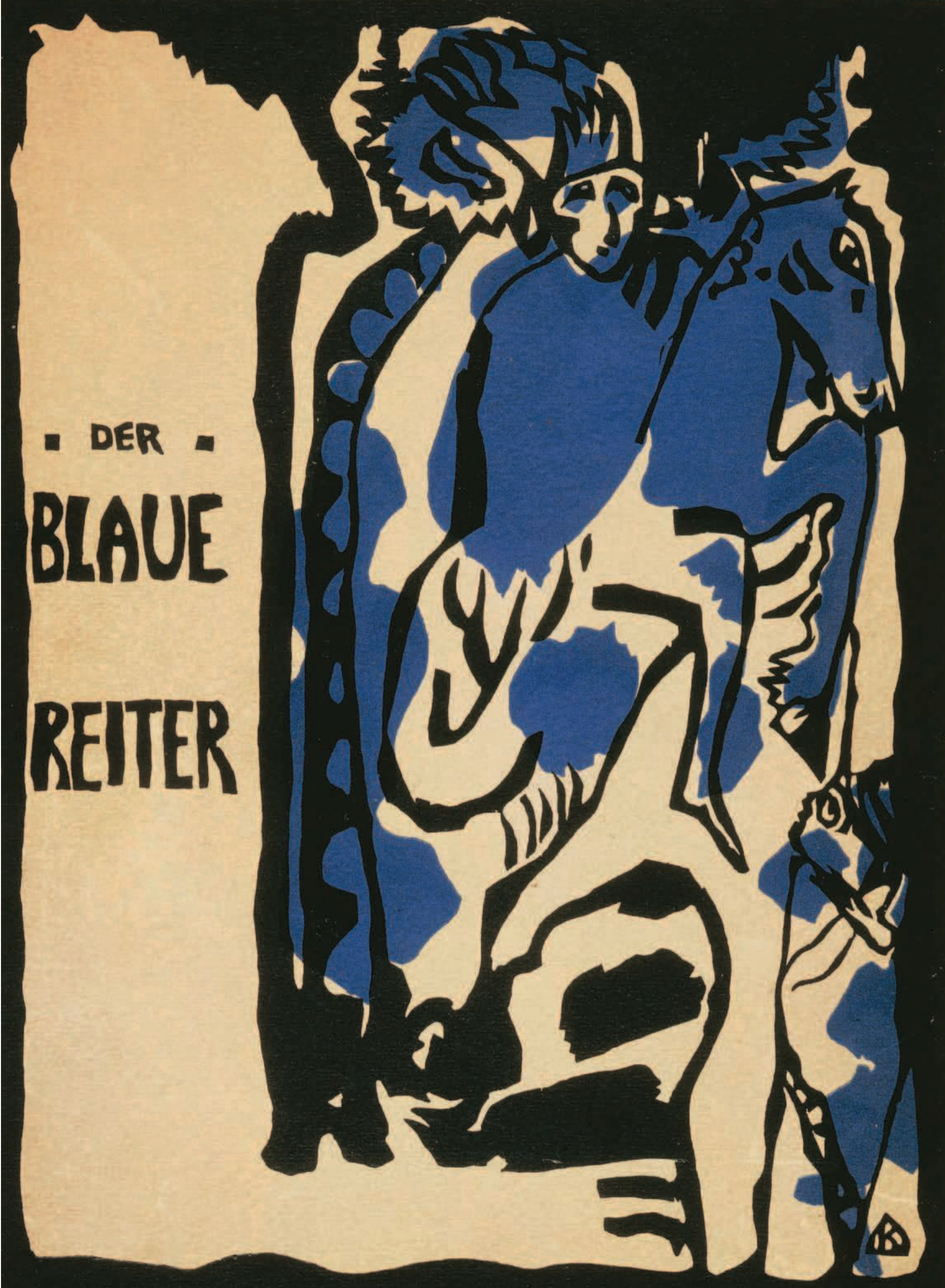




\section{Le Cavalier bleu}

Aux alentours de 1911, Franz Marc (né en 1880, mort à la guerre en 1916), Vassily Kandinsky et Paul Klee formèrent une communauté, le «Cavalier bleu ». Le sens et les intentions de ce groupe furent présentés avec force en 1912 dans le recueil éponyme. Ce groupe, qui n'était pas une association, cette fois, mais une communauté spirituelle, m’apparaît comme le cœur de l'histoire de l'art allemand contemporain.

Ce qui a été expérimenté et découvert dans ce groupe était autre chose qu'une variante de l'artisanat pictural. Un point décisif est que, pour ces gens, il en allait de quelque chose de plus important que la seule peinture, à savoir d'une transformation de la structure psychique. L'histoire de ce mouvement coïncida exactement avec l'agonie de la grande peinture européenne. Enfin, les Allemands posaient le problème de l'autonomie de la peinture et du libre développement de processus hallucinatoires.

Il ne s'agira pas ici de présenter une critique de chaque peintre pris individuellement, ni d'examiner comment les problèmes ont été traités individuellement par chacun d'eux; le Cavalier bleu était une communauté d'hommes qui assignaient à l'art allemand un objectif tout à fait nouveau; ils avaient compris que la peinture, si elle possédait une raison d'être, devait être autre chose et davantage qu'un pur artisanat et un exercice d'adresse pour singes très savants.

L'expressionnisme, qui n'avait pas dépassé la lâcheté de l'ornementation, avait accepté l'ordre établi et s'était contenté de variantes indigentes, était mort. Ce qui se faisait à l'époque dans la peinture allemande en dehors de ce groupe nous apparaît comme un vieil artisanat exsangue. Bien entendu, des réactions se manifestèrent plus tard contre l'esprit du Cavalier bleu, par exemple au nom des lâches conforts de la nouvelle objectivité et de sa ringardise; un slogan était censé masquer son manque de personnalité et d'imagination. Mais cette affectation d'objectivité n'était qu'une réaction et une vengeance de petits-bourgeois obsédés par la propriété, qui ne pouvaient tolérer la conquête d'une liberté intérieure.

Marc, Kandinsky et Klee tentèrent avec moins de retenue que les autres Allemands d'introduire dans la peinture une attitude humaine différente. Enfin, on avait le courage de détruire les consensus dépassés et inhibants; enfin, on osait dessiner sans réserve les visions qu'on avait découvertes. On cherchait à fixer la vision intérieure dans sa dimension de processus, on dynamisait l'image, afin que des événements visionnaires fussent directement à l'œuvre dans le tableau. Le peintre n'était plus là pour représenter ni pour arranger, il n'était qu'un pur médium des visions. On combattit l'inertie statique des toiles classiques, afin de retranscrire de façon plus désinhibée des processus jusque-là demeurés à l'état inconscient.

Rarement une révolte aussi absolue avait secoué l'Allemagne. On ne réclamait pas de nouvelles images, mais autre chose: de nouvelles dispositions psychiques. La conception de l'artiste était ainsi étendue bien au-delà de la compétence artisanale.

L'histoire de ce groupe est proprement allemande. Des jeunes gens se lient, la guerre les sépare. Franz Marc meurt au front; Kandinsky part en Russie; là-bas, il vit la révolution. En Russie, dans un premier élan, on espérait trouver des solutions globales. Cette situation convenait assez bien au dogmatisme un peu vague d'un Kandinsky. L'homme des visions isolées tente désormais de tirer une théorie et une construction doctrinale des expériences qu'il a vécues. On aspire à mettre au point une technique d'hallucination susceptible d'être transmise; il faut enseigner aux élèves la vision intérieure. Un entraînement de derviches, ou quelque chose d'approchant. Après la guerre, Kandinsky retourne en Allemagne et retrouve Klee au Bauhaus.

Dans le Bauhaus - qui était en fin de compte une école d'artisanat, mais fondée sur des bases différentes -, on voulait s'exercer à acquérir une âme nouvelle, et le faire à fond, à l'allemande. Un objectif qui convenait mieux à l'activisme étroit des constructivistes qu'à ceux qui vivaient des expériences vraiment inédites.

En Russie, Kandinsky avait sans doute été influencé par les suprématistes. Les processus politiques, là-bas, s'étaient déroulés conformément à la prophétie scientifique de Karl Marx, et l'on s'était mis à croire, par suite, que la science était suffisamment avancée pour se prononcer sur toutes les questions. C'est ainsi que, chez les 


\section{TEXTES DE CARL EINSTEIN}

artistes russes, la foi dans la doctrine s'était considérablementrenforcéeet avaittrouvéune confirmation.Une orthodoxie du nouvel objet. Une religiosité tournant à vide s'empara alors de la théorie de l'art, qui était faible. Cependant, comme il était impossible d'aborder en quelque façon des phénomènes psychiques complexes, on se contentait de doctrines indigentes sur le carré et le triangle. On pratiquait une esthétique qui était du niveau de la psychologie associative ou atomistique des années 1890 et on philosophait sur les éléments au lieu d'étudier des phénomènes psychiques complexes.

Les effets de ce type d'attitude vieillotte, qui évoquait, en gros, l'époque des Lumières, se manifestèrent chez Kandinsky, à son retour. Ce peintre, qui s'était précédemment illustré par l'attention qu'il portait aux processus, à la dimension dynamique, était devenu un géomètre qui jouait, même s'il le faisait avec beaucoup de goût. Avec le triangle et le cercle, la forme libre s'était muée en une symbolisation pédante.

Franz Marc mourut à la guerre, et nous ne possédons de lui qu'une œuvre naissante. Ses dessins de guerre attestent que cet homme avait continué à évoluer, même si ces œuvres recèlent pas mal de banalités et de fatras cosmiques qu'il vaut mieux oublier.

Quant à Paul Klee, c'est un fait : il réussit à dépasser les problèmes esthétiques et à inventer de nouveaux objets, à introduire dans la réalité les figures qu'il forgeait intérieurement et à concurrencer ainsi cette dernière.

Voilà pourquoi nous voyons en Klee la personnalité la plus remarquable parmi les artistes allemands. Des kilomètres de toiles ne suffiront pas à nous faire changer d'avis : les miniatures de Klee valent mieux que les monuments de peintres académiques survoltés, pathétiques torchons.

Les deux artistes du Cavalier bleu qui sont encore en vie ont aujourd'hui entre cinquante et soixante ans. Hélas! Nous n'avons d'autre choix que de les compter parmi les jeunes. C'est dire si la question de la relève est problématique. Preuve qu'on a tort de tenir pour " jeune » une peinture simplement parce que son auteur appartient, dans les chiffres, à une génération plus récente. La jeune génération est souvent réactionnaire et défend l'héritage des grands-pères contre les révoltés de la précédente. Ainsi, pour nous, les jeunes de la nouvelle objectivité sont des réactionnaires sans couleur. La peinture de Die Brücke est l'équivalent de celle des fauves, cela reste une peinture postimpressionniste dans l'esprit de 1908 et 1910. Beckmann et Grosz incarnent, selon nous, une réaction contre les insuffisances de l'expressionnisme; et sans doute aussi, dans le cas de Beckmann, une certaine réaction contre la peinture dite «abstraite». En revanche, nous situons Kokoschka dans le postimpressionnisme; en d'autres termes, la position de Beckmann est intellectuellement plus complexe que celle de Kokoschka.

Chez Klee, cependant, nous n'avons pas affaire à une peinture abstraite; c'est bien plutôt l'inverse, justement, il introduit des figures et des objets nouveaux, non pas en opérant des combinaisons, mais en se fondant sur la libre invention de processus psychiques. Klee exploite des phénomènes psychiques jusque-là ignorés dans la peinture allemande du xix ${ }^{e}$ siècle. C'est surtout en cela que cet homme nous paraît remarquable. Nous sommes d'avis, en revanche, que l'analyse d'un Beckmann ne livrera guère d'éléments nouveaux, décisifs d'un point de vue humain.

$\mathrm{Au}$ final, ce qui compte dans le cours de l'histoire n'est pas la répétition mais la destruction du donné et l'élaboration du nouveau, bien que les efforts fournis à des fins de conservation l'emportent, et de loin, sur la capacité à produire effectivement du neuf - un constat qui nous fait croire à tort que l'art est ni plus ni moins réactionnaire. Je suis conscient que le regard que je porte sur l'effort imaginatif en Allemagne comme en France s'inscrit dans une perspective de proximité; plus tard, on repérera des stades intermédiaires et des passages; c'est là le résultat de l'adaptation, de part et d'autre, de l'image et du monde.

Le point important, concernant le groupe du Cavalier bleu, reste que, enfin, des peintres ont affronté le problème du rationnel et de l'hallucination. On a adopté un parti pris romantique, à savoir qu'on a cessé d'idéaliser les solutions en apparence élégantes et astucieuses de la raison bornée pour ouvrir des domaines psychiques qui vivotaient jusque-là dans les bas-fonds maudits et obscurs de la superstition et de l'informe.

Comme la jeune génération l'a fait plus tard en France, on a pris le parti du romantisme et de la représentation de processus libres, intérieurs.

Kandinsky est retombé dans le didactisme et la rationalité géométrique. Dès ses débuts, il croyait à une sorte de physique sentimentale des couleurs; une série d'allégories était censée tenir lieu de connaissance. Au demeurant, l'effort dynamique fut limité de manière regrettable, parce que l'analyse se conformait à une psychologie complètement dépassée et que des méthodes statiques de la psychologie étaient appliquées à des processus complexes auxquels elles n'étaient pas adaptées. 
Klee, quant à lui, a abandonné les sphères bornées de l'indifférence esthétique et s'est lancé courageusement dans la réalisation de formes. Voilà pourquoi nous voyons en lui le représentant majeur du nouveau romantisme allemand.

Les artistes du Cavalier bleu se sont défendus contre leur isolement hautain en cherchant à universaliser, à l'aide de méthodes intellectuelles vieillies, leurs expériences particulières, qui deviennent des types. Dans leurs livres, ils ont décomposé les processus psychiques en différents éléments, au lieu d'exposer le complexe unique d'un vécu dans sa totalité et de construire une typologie des phénomènes psychiques à partir de cette base. Avec un tel ersatz de connaissance, on cherche justement à se protéger contre la violence de l'imagination; on croit pouvoir généraliser des expériences vécues en distinguant, dans leurs résultats, des éléments abstraits et universaux. Pourtant, la pure ligne droite ou la pure couleur ne sont pas la condition d'une expérience; elles en sont la conséquence, détachée du vécu et généralisée. Les livres de ces artistes ont conforté point par point les clichés éculés des théoriciens de l'esthétique, qui ne sont pas en mesure de rendre compte des expériences vécues et se contentent, à la place, de comparer dialectiquement entre eux des concepts presque dépourvus de contenu. Il est vrai que l'on peut invoquer, à la décharge de ces artistes, une situation de légitime défense face au tumulte du vécu.

\section{Franz Marc}

L'expressionnisme nous apparaît comme un intermède, un armistice. On transpose certains schèmes formels sur les choses et on cherche à prouver ainsi sa maîtrise sur celles-ci.

Un instrument de pouvoir utilisé à cette fin est la réduction du motif à une surface. On commence par priver le motif de sa corporéité et, par là, de sa force active. Lorsque l'homme crée une forme, il se venge du monde qui l'entoure : celui-ci l'oppresse et il le craint d'autant plus qu'il entrave ses capacités psychiques d'invention libre et autonome.

Nous commencerons par évoquer Franz Marc parce qu'il fait le lien entre l'expressionnisme et le Cavalier bleu. Dans ses tableaux, Marc reste toujours attaché au motif, et celui-ci revêt chez lui une importance particulière.

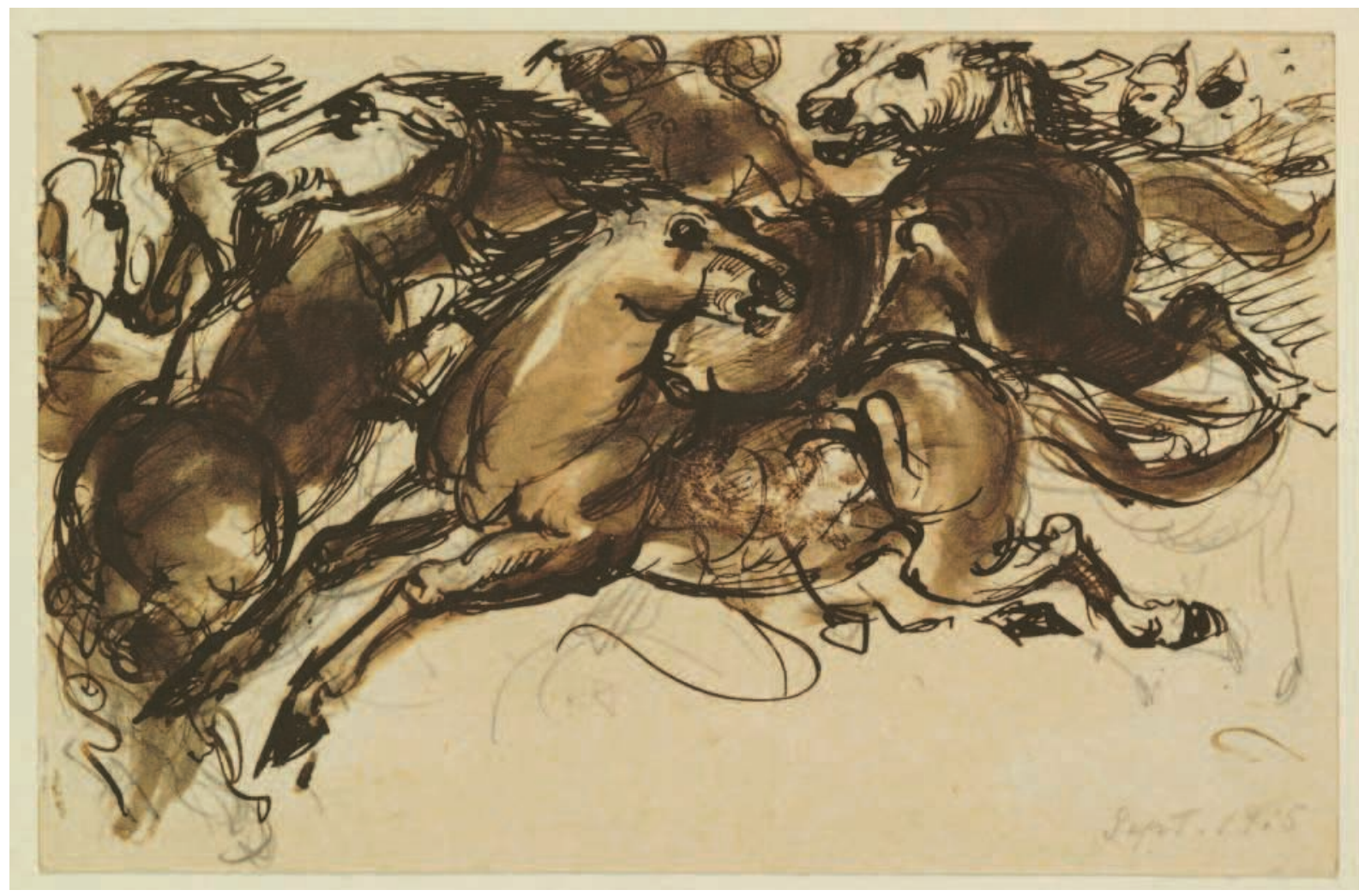

Fig. 5 Franz Marc, In wildem Lauf dahin sprengende Pferde, 1915 ๑ BPK, dist. RMN / Kupferstichkabinett, SMB / Reinhard Saczewski. 


\section{TEXTES DE CARL EINSTEIN}

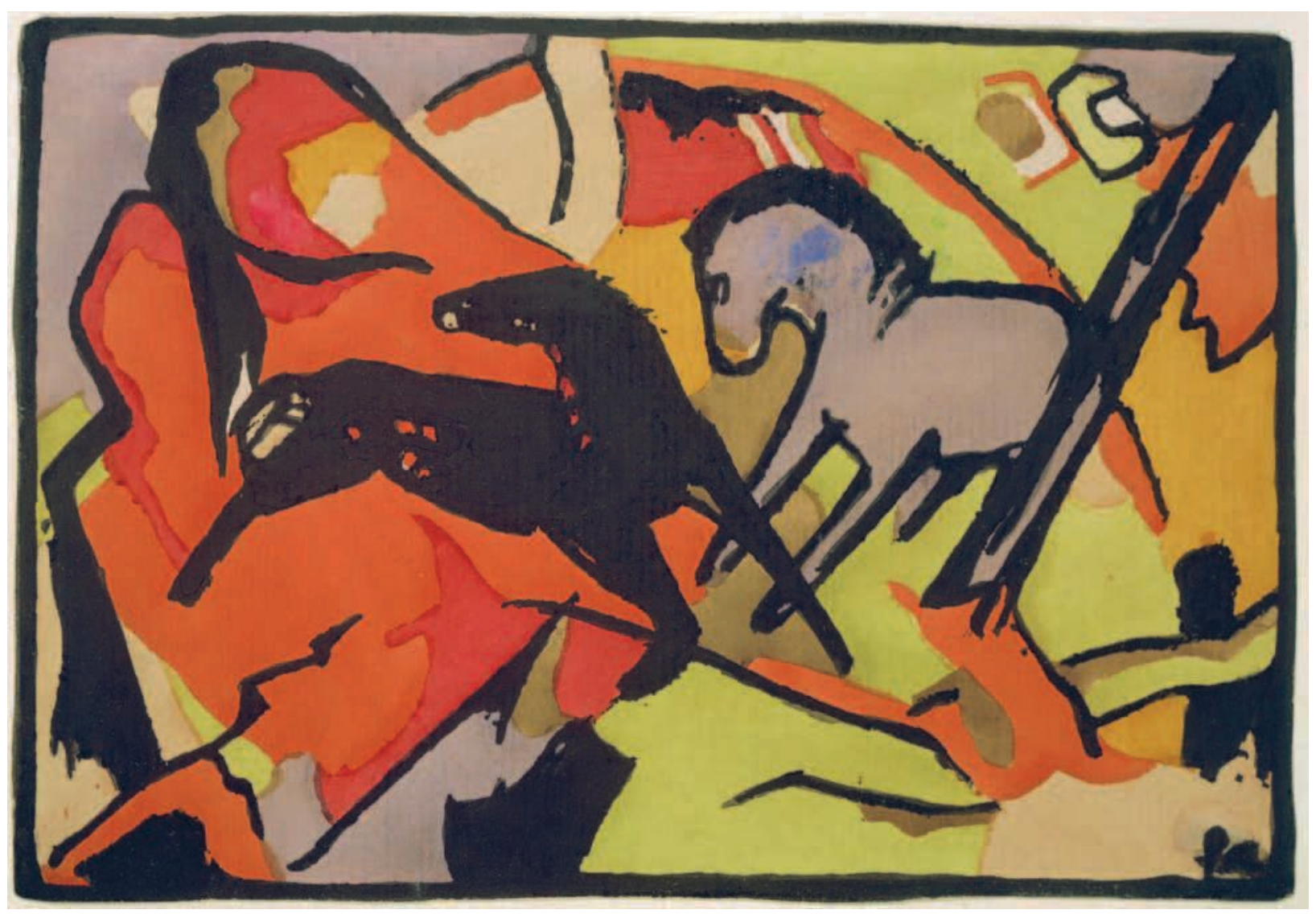

Fig. 6 Franz Marc, Deux chevaux, 1911-1912 @ BPK, Berlin, dist. RMN / Elke Walford.

L'important reste que, dans le Cavalier bleu, les aspirations introverties sont vécues et assumées plus librement. Elles sont nécessaires à l'homme pour qu'il s'affirme en face de la nature dont il subit l'action de façon pénible. Il est ainsi contraint de renforcer l'antagonisme qui l'oppose à celle-ci. D'où un conflit. On ne se retrouve plus dans les choses. On est à la marge du monde et on croit que «l'esprit peut vivre sans corps». On cherche alors, comme l'écrit Marc, une «expression pure " de l'âme, "étrangère à toute image du monde ", on croit à «la bénédiction de la mort, à la destruction de la forme, afin que l'âme soit libérée "; on rejette l'individuation dans les choses, on ne recherche pas le "cas personnel et singulier " mais, en somnambule, on se concentre sur le typique, sur «la contemplation de rapports de tension contraignants». On ne veut pas peindre des souvenirs mais poser ce qui est à venir, «l'esquisse d'un monde nouveau ".

On cherche à imaginer un équilibre entre la science et l'art, on veut " percer à jour les choses " pour découvrir un arrière-plan «que les choses, avec leurs apparences, ont plutôt tendance à dissimuler ", en "faisant croire à l'homme qu'elles recèlent davantage que ce qu'elles renferment effectivement». L'art doit emprunter la voie de la science, affirme-t-on, dévoiler les éléments les plus vrais de tout phénomène, "afin de mettre au jour et d'exposer les idées pures qui sont au fondement de l'édifice du monde », "de séparer l'essentiel de l'inessentiel » et « de prendre part au royaume de Dieu, à l'Esprit saint». L'abstrait apparaît «comme le voir naturel, comme la vision intuitive première", qui permet de saisir, "par la troisième vue, une formule de toutes les lois", et de ne plus peindre ainsi la représentation des choses mais leur volonté même. L'art débouchera alors sur un « monde de symboles nouveaux " et on vivra " au milieu de visions étrangères, d'images nouvelles et de sonorités inouïes ». "Il faut repenser l'homme dans sa totalité ", et la peinture est une tentative pour échapper à la «douleur de l'absence de forme». L'art à venir sera «le devenir-forme de notre certitude scientifique; il est notre religion, notre point fort, notre vérité». Auparavant, l'au-delà revêtait, dans l'art, les formes 
du monde visible. Aujourd'hui, nous ne rêvons plus en nous laissant borner par les choses, nous les nions, car " notre savoir s'est frayé un chemin jusqu'à la vie qu'elles recèlent». Ce "savoir encore latent aujourd'hui se transformera demain en une force créatrice de formes plastiques »; car «la science n'est pas une fin, c'est une modalité de notre esprit». Le "problème religieux du contenu » sera au centre de cet effort pour créer des formes. On donnera forme «à la détermination intérieure des choses ", à leur "volonté", non à des masques. Une formule proprement schopenhauérienne.

On veut échapper à «l'étroite obsession du moi"; à la vanité du "cas personnel et singulier», on oppose l'intuition que «quelque part, au point le plus profond, les hommes peuvent être égaux ». Voilà pourquoi on est un artiste primitif. Il est significatif que l'importance des maîtres anciens, la proximité à leur égard, soient perçues de façon presque négative; on valorise surtout chez eux le rejet de la perception, leur sens pur qui n'est guère subordonné au phénomène. Comme eux, on veut créer des images impersonnelles, et ne pas les amoindrir par la vanité d'une signature.

Une vision est ici créée qui va bien au-delà de la perception, pleine de pessimisme à l'encontre de l'individuel. Tout acte de foi trouve son pendant dans un scepticisme résolu à l'encontre de ce à quoi on n'accorde pas foi. La valorisation de l'intériorité implique une déception par rapport au monde qui se donne à voir; on s'enferme en soi-même. Il est un point sur lequel ces peintres s'écartent des platonismes des maîtres anciens : ils ne cessent de mettre l'accent sur la dimension de processus inhérente à la représentation plastique. Marc écrit beaucoup sur cette question; selon lui, il faut "faire parler le monde même"; il se demande comment on peut "peindre l'être d'un chien", comment Picasso peint l'être d'une forme cubique. Ouand on crée, "le chevreuil éprouve ", c'est-à-dire le prédicat, tandis que Pisanello, lui, peignait le chevreuil (le sujet); pour Marc, «le paysage doit être chevreuil »; autrement dit, on veut échapper au subjectif en disparaissant entièrement dans le vouloir de ce qui est représenté, si bien que, dans la contemplation, sujet et objet, artiste et motif ne font plus qu'un. On reconnaît ici des tournures schopenhauériennes, des conceptions qui rappellent l'Inde: "Tu es moi." La subjectivité pleine touche à son contraire dialectique et, pour finir, recèle directement son objet comme une force dynamique, au lieu de l'éprouver comme un poids mort. Par cette union, dans la contemplation, avec l'objet représenté, on croit pouvoir éprouver celui-ci dans sa pureté, en mettant entre parenthèses sa propre volonté; c'est le chevreuil qui éprouve à travers mon moi métamorphosé. La subjectivité, dans son point de tension maximale, aboutit, sur un mode bipolaire, à une métamorphose du moi; le problème du contenu se pose ainsi avec acuité. Dissolution du sujet dans un contenu dont on croit percevoir l'essence pure parce qu'on s'est soi-même réduit à néant dans la volonté du contenu. "L'art est métaphysique, le sera, ne peut l'être qu'à partir de maintenant. L'art va se libérer des finalités humaines et du vouloir humain. Nous ne peindrons plus la forêt ou le cheval comme ils nous plaisent ou comme nous croyons les voir, mais comme ils sont effectivement, comme la forêt et le cheval s'éprouvent eux-mêmes, leur essence absolue qui vit par-delà l'apparence à laquelle se limite notre vision... Toute création artistique est alogique. Il existe des formes artistiques qui sont abstraites; [...] il en a existé de tout temps, mais elles ont constamment été brouillées par le savoir humain, le vouloir humain. La foi dans l'art... vit de l'autre côté. »

Franz Marc veut entrer, par l'extase, dans l'émotion vécue des animaux, il oppose à l'interprétation subjective de l'art contemporain la disparition du moi dans l'émotion vécue des contenus représentés. $\mathrm{Au}$ demeurant, il assigne une limite à cette extase du peintre: «Le seul prédicat que l'on donne, c'est celui de la nature muette; comment donner le prédicat de la nature vivante, c'est un problème qui n'est toujours pas résolu. »Cette façon de voir le sépare des futuristes. Cette fusion religieuse dans des contenus objectaux, cette rupture résolue avec l'isolement subjectif éloignent Marc de Kandinsky; ses visions cosmiques oniriques le rapprochent de Klee. Mais celui-ci invente des objets fantastiques en adéquation avec le sujet, tandis que Marc parie sur un renforcement de la tension : une disparition dans l'image d'un animal ou des étoiles. La dimension centrifuge de la forme contemporaine est élargie à l'échelle cosmique. Au final, le monde est entièrement vécu dans la forme subjectivement pure et ce sujet affranchi de tout sentimentalisme de l'individualité, qui vit les choses comme un pur processus, peut réaliser une union dynamique avec ces dernières et épouser leur mouvement.

Marc abandonne la représentation humaine et son " étroite obsession du moi " et peint des animaux - « non comme je les vois mais comme ils sont (comme euxmêmes voient le monde et ressentent l'être de celui-ci) ". L'instinct le conduit à aller de l'émotion vécue en face de l'homme à l'émotion ressentie face aux animaux purs. L'homme impie n'éveille pas en lui les sentiments vrais, 


\section{TEXTES DE CARL EINSTEIN}

c'est l'émotion vécue de l'animal, intacte, qui fait écho à ce qui est bon en lui. «Et un instinct me détourna de l'animal pour me mener vers l'abstraction, [...] vers la deuxième vision, qui est entièrement indienne, intemporelle... Très tôt, j'eus le sentiment que l'homme était laid; l'animal me semblait plus beau, plus pur; mais chez lui aussi, je découvris tant d'aspects répugnants et hideux que mes représentations devinrent instinctivement plus schématiques et plus abstraites. » Il perçoit toujours plus vivement «la laideur de la nature, son impureté " et cherche dès lors "une expression pure, étrangère à toute image du monde ». Il dit quelque part qu'il s'est «détourné de toutes les grimaces » et que ses tableaux sont des «dialogues avec lui-même ».

Ces représentations animales ont pour fondement un pessimisme ascétique chrétien. Peindre les créatures pures, c'est déplorer la dépravation de l'homme; mais la nature elle-même est impure, et on la fuit, pour puiser dans son intériorité des visions toujours plus distanciées, des idées pures. Le péché originel pousse à aller vers l'abstraction; mais possède-t-on soi-même la pureté de l'esprit? Chez Kandinsky et Marc, on observe, pourrait-on dire, une position mystique de rejet de l'optique; on peint, mais les sens sont tout entiers voués au péché et à la corruption; on cherche à contempler les sphères les plus pures de l'esprit intérieur et on plane sur les sommets du «rien » optique; dangereuse réduction de la vision par contrainte métaphysique; amoindrissement du monde au nom de la pureté.

Il faudrait écrire un jour la psychologie de ces motifs picturaux. On ne sait si, en représentant le paysage, l'homme domine désormais la nature ou bien lui est soumis. Peut-être pouvons-nous voir là une projection de l'homme dans le monde qui l'entoure, une fuite devant lui-même et une métamorphose en ce qui lui est étranger. D'un point de vue psychique, on se délivre d'un poids. L'attachement de Marc au motif de l'animal est singulier. Il joue le drame de la métamorphose. Se transformer en animal, c'est délivrer l'homme d'acquis de la civilisation qui sont autant d'inhibitions et de falsifications; c'est se primitiviser et fuir l'homme. L'animal apparaît ici comme un stade intermédiaire, une étape dans la migration des âmes, pour s'éloigner de l'homme. Peut-être pouvons-nous deviner ici un atavisme légendaire.

Nous représentons des choses extra-humaines pour les dominer, certainement, mais aussi pour échapper au standard monotone du corps humain. La figure humaine est devenue insuffisante pour exprimer nos sensations. Marc reste fixé sur l'animal. Ses esquisses de la guerre en témoignent : nul doute qu'il aurait plus tard élargi son univers. Mais dans l'œuvre existante, il reste attaché à l'ancêtre animal, sur un mode totémique. Il faudrait étudier quelles impressions d'enfance furent déterminantes dans la genèse d'une telle fixation.

Marc écrivit un jour qu'il regardait la nature «avec un sentiment de compassion, une sorte de savoir partagé; [...] nous nous comprenons bien, la vérité est vraiment ailleurs; nous venons tous d'elle et retournerons à elle un jour ". Ainsi, en un sens, la nature est encore pour lui un costume, un masque. En esprit, il voit «à travers les choses la pure ligne de la pensée » et il avoue à regret: "Je ne suis presque jamais parvenu à faire le lien entre celle-ci et la vie, du moins la vie humaine (voilà pourquoi je ne peux peindre des hommes).»

Chez Marc, le rejet de l'anecdote et de l'individuel s'incarne dans l'animal docile, qui est plus éloigné de la multiplicité des individuations et sert à porter des formes plus libres. À l'isolement induit par la forme subjective, on oppose l'amour pour l'animal sans défense, contenant docile; contre l'isolement lyrique de la pensée, on réagit par une intuition cosmique de la proximité des animaux et des plantes, tentative audacieuse pour figurer directement les sensations typiquement animales. Cette tentative pour fixer la dynamique prédicative par la métamorphose cosmique du moi est une réaction de défense contre l'isolement mortel dans lequel entraînent les pures idéologies, qui menacent la composante optique, plus faible. Instinctivement, Marc cherche à neutraliser le christianisme à la manière franciscaine; car nous avons perdu le confortable et multiple équilibre qui caractérisait la relation des Anciens avec le monde; pour eux, le spirituel était présent en chaque chose sous une forme corporelle, incarnée, sa vérité était tangible à bien des égards, il était isolé et figuré dans chaque pan du réel.

Un artiste qui endure le mystère effréné et sévère de la pureté. À cette destruction ascétique de l'être correspond le démantèlement de la forme au profit d'un contenu éprouvé comme religieux. On a presque honte de poser la question du comment. On a envie de dire que ce type de peinture, qui avait déjà périclité à force de descriptions scientifiques, de singeries anecdotiques et historiques, était une nouvelle fois menacé par la peinture anecdotique et historique de l'esprit pur, de l'abstraction. Il est facile de se perdre dans les philosophèmes du sentiment, dans une sorte de théosophie, et il reste alors trop peu de forces pour le visible. Ce prologue merveilleux use trop de forces et la plastique s'amoindrit quand la métaphysique prend de l'ampleur. 


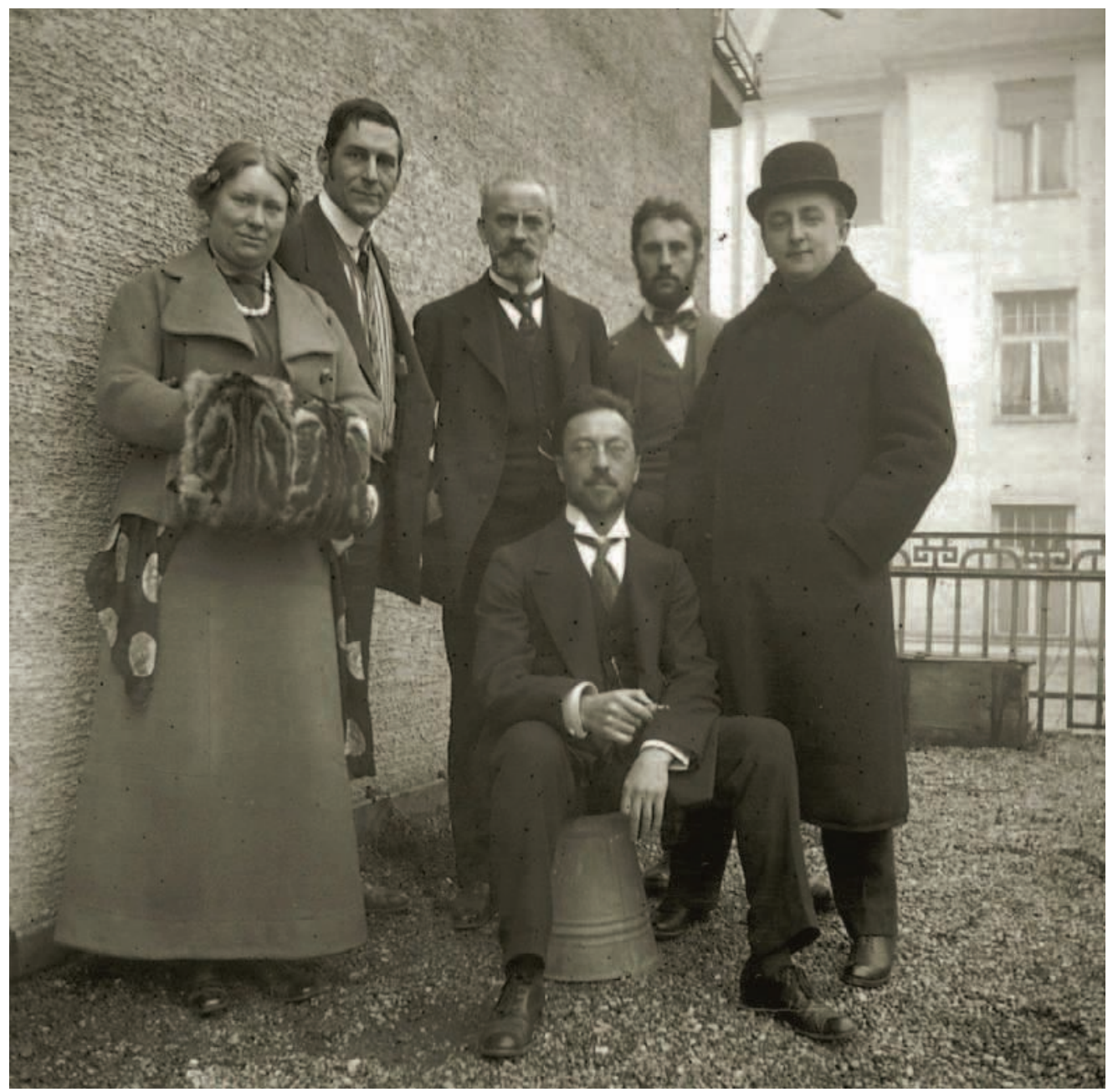

Fig. 7 Marie et Franz Marc, Bernhard Koehler, Vassily Kandinsky, Heinrich Campendonk, Thomas von Hartmann, Munich, 1911-1912, collection Gabriele Münter et Johannes Eichner, Fondation de Munich.

Marc songeait souvent aux maîtres anciens et à leur pureté. Il écrivait au demeurant: «Les tableaux purs sont si rares »; il n'en tirait pas la conclusion que son intériorité, sa pureté étaient peut-être hostiles à la majeure partie de la peinture en général, et que l'accomplissement spirituel qu'il visait ne pouvait être atteint qu'au prix d'une partialité désespérée. Sa mystique sensible était éloignée du monde de ces primitifs, qui avaient hérité de la religion et de l'Église beaucoup de visions - peut-être païennes. Les primitifs vivaient dans un monde spirituel dont la forme était préétablie. Les peintres comme Marc et Kandinsky voulaient, eux, tirer un tel monde de l'immédiateté du moi et s'épuisaient d'emblée dans le prologue spirituel. Ils ne disposaient pas d'un univers religieux structuré, d'un monde ordonné de symboles, et ils s'enfermaient ainsi dans une mystique égocentrique, parce qu'ils voulaient interpréter les processus psychiques à l'aide des clichés statiques d'une science physique dépassée.

Kandinsky resta pris dans le lyrisme subjectif, qui allait dans le sens du décoratif; Marc compensait cet égoïsme religieux en cherchant à se métamorphoser dans la pure essence de l'animal. L'homme est «un produit de transition, comme l'animal et la plante ", et il est capable, par un acte visionnaire, de retourner à l'animal. On retrouve là des motifs indiens et l'éternel retour de Nietzsche. Marc se demandait comment les forêts se reflètent, le soir, dans l'œil du chevreuil, et les eaux scintillantes d'un étang dans le regard de la poule d'eau. Il voulait constamment saisir la dynamique immédiate de l'objet. Il pensait que les cubistes étaient parvenus à le faire; en quoi il avait tort; car ces derniers donnaient forme à la plénitude événementielle du volume à partir du mouvement de leur propre regard. 


\section{TEXTES DE CARL EINSTEIN}

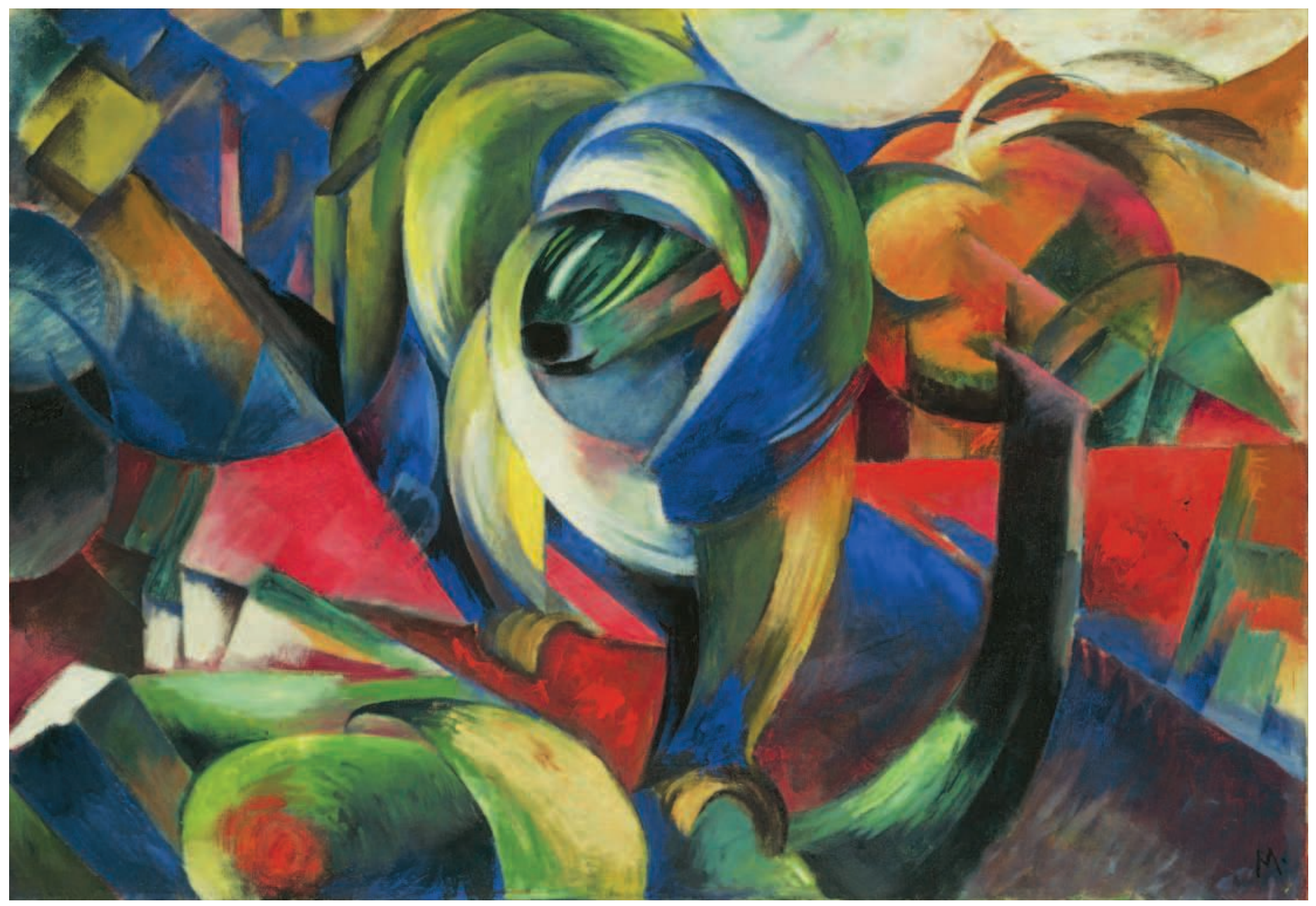

Fig. 8 Franz Marc, Le Mandrill, 1913, Munich, Pinakothek der Moderne, collection Moderne Kunst @ BPK, Berlin, dist. RMN / image BStGS. 
Ce qui faisait défaut à Marc, comme à Kandinsky, c'était un contrepoids à leur mystique dévoreuse de la forme. Dans leurs tableaux, le vide et le ravissement extatique masquent la faiblesse et l'approximation de la forme, et l'abstraction métaphysique tourne un peu à vide en allant vers l'ornement et le décoratif.

Comme Klee, Marc retint les leçons des cubistes; il avait recours, de manière un peu pathétique, à leur mode de stratification de la forme, tout en restant pris dans un cubisme de stylisation instinctive. Dans la pensée, on s'était détaché du monde - en suivant des voies anciennes-; dans la peinture, on hésite longtemps entre forme imaginative et objet traditionnel; le résultat est une dérive pathétique vers l'ornemental. Le monde est peut-être dépassé dans la réflexion, mais la forme interne est trop faiblement constituée. Cet héroïsme vire à la négativité; à force d'extase, on sombre dans l'ornemental, et l'organisation de l'espace est caricaturalement cubiste; la métaphysique avait vidé le regard et n'autorisait plus que le jeu de couleurs d'éléments optiques trop pauvres. Marc avait la pureté des êtres qui doivent anéantir la nature et leur propre nature. Ses yeux affaiblis par la quête de l'absolu se perdent dans des ornements : l'élan de mise en forme n'est pas encore suffisamment affirmé. Une foi monumentale fait surgir brusquement une première forme, mais il lui manque un contrepoint maîtrisé; le sentiment cosmique et l'extase de l'absolu s'achèvent tragiquement dans une tentative pathétique, un peu mécanique.

C'est justement parce que nous portons un tel jugement que nous avons laissé la parole à Franz Marc, qui mourut jeune, afin qu'il interprète et défende lui-même son œuvre.

\section{Vassily Kandinsky}

Autour de 1910, l'expérience à laquelle les peintres furent confrontés était la nécessité de tirer les conséquences du dualisme opposant un processus interne toujours plus isolé et un héritage devenu mécanique. On aboutit nécessairement à des formes qui, désormais indépendantes des choses, possédaient une sorte d'existence propre. Les impressionnistes avaient laissé une œuvre très accommodante de ce point de vue. Peu à peu, ces formes imaginatives furent adaptées par le bourgeois; vers 1920, au terme d'une jeunesse héroïque dont le parcours avait été monologique, on s'efforça à nouveau de saisir la réalité tangible; les expériences de jeunesse furent banalisées et appliquées au donné; car

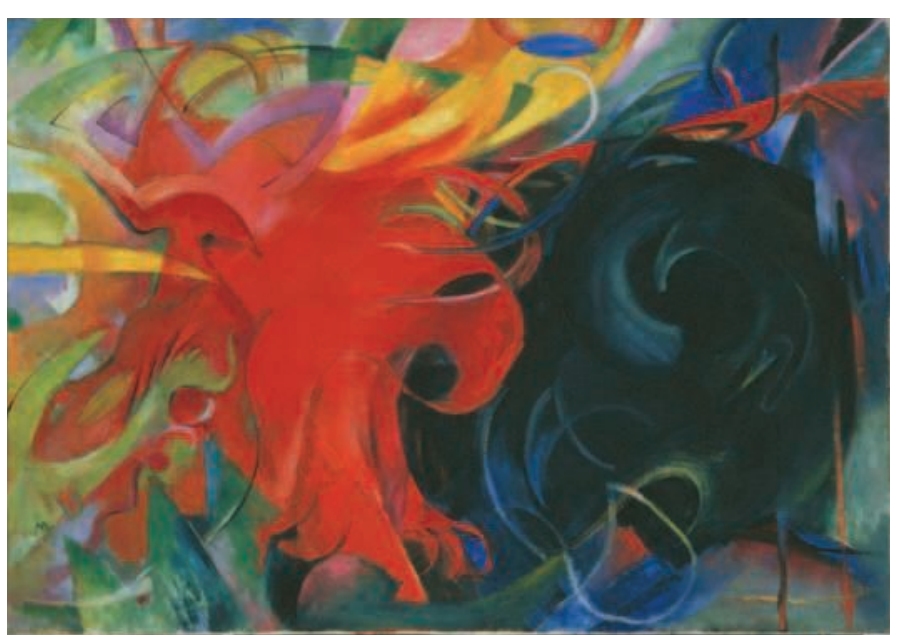

Fig. 9 Franz Marc, Formes combattantes, 1914, Munich, Pinakothek der Moderne, collection Moderne Kunst @ BPK, Berlin, dist. RMN / image BStGS.

notre homme ne se satisfaisait guère du cercle étroit du monologue lyrique. Il s'agissait de maîtriser les choses et les hommes, on cherchait désormais à projeter sur la terre le dédoublement visuel, à conquérir le donné grâce à ce dernier et à se placer soi-même dans une position de maîtrise directe des choses. On avait recours à des compromis trompeurs dans lesquels il était facile de tomber. Certains furent poussés jusqu'au vérisme photographique; entre 1914 et 1920, en effet, la réalité était devenue plus pénétrante que jamais.

Le Russe Kandinsky fut confronté à large échelle à un dualisme du motif et du contenu interne; il sentait que le motif observé ne délimitait pas directement le processus psychique, qui était perçu non comme un accident contingent mais comme le noyau irréductible des événements. On se coupait de l'extérieur et on se repliait sur le moi nu, qui explosait en couleurs comme par l'effet d'une nécessité. Pour certains, ce renversement était le produit d'une incapacité à peindre, à quoi on peut répliquer que les mystiques ne font pas de bons reporters et que, a priori, leur talent ne peut être mis en doute.

C'était là l'expérience vécue: la séparation entre l'objet, contingent, et le contenu intérieur qui en était indissociable, ressenti, lui, comme nécessaire. Par là, la question de l'autonomie des processus intérieurs était soulevée, et, avec elle, le problème de la liberté humaine. Kandinsky établit une opposition dualiste entre le contenu intérieur, nécessaire, et l'objet, donné de l'extérieur, et tenta de démontrer que des formes hallucinatoires spécifiques correspondaient au sens intérieur de la forme. Les choses étaient pulvérisées sous l'effet d'un pur solipsisme optique : ruine de l'objet. 


\section{TEXTES DE CARL EINSTEIN}

Kandinsky était né en 1866 à Moscou. Jamais il n'oublia cette vieille ville et ses couleurs. Il suivit une formation de juriste et travailla sur le droit des paysans de la vieille Russie. Il fut frappé par le fait que les paysans russes, dans les procès, ne mettaient pas en avant les faits mais les dispositions psychiques de l'accusé. Vers 1900, il partit pour Munich et devint peintre. Pendant la guerre, il retourna en Russie. En 1921, il revint en Occident.

Kandinsky trouva peu à peu sa voie, après des débuts insignifiants. Dans ses tableaux, il échouait le plus souvent en se heurtant au concret; insatisfait devant les choses, il cherchait à contourner ces dernières en se réfugiant dans une poésie évocatrice ou dans le merveilleux; il ne sut guère éviter de tomber dans ce que la jeune peinture munichoise avait de plus discutable. La force immédiate de la couleur s'impose toujours davantage à lui : en 1909, il peint sa première improvisation, en 1910 sa Composition I. Le motif et la forme intérieure de la couleur sont encore liés; il forge pour cela la catégorie des "impressions", ou désigne sous le nom de "poésie» ces options qui restent encore déterminées par un objet. En 1911, il peint sa première peinture «abstraite».

Kandinsky fut à l'origine d'une révolte emblématique d'une génération : la rupture avec les choses, le désir de peindre un événement psychique dans son immédiateté et sa nudité. On méprise l'expression métaphorique, on aspire à l'immédiateté du moi et, par suite, on se détourne du monde des choses reproductibles; ces artistes sont saisis par l'angoisse que les choses ne soient que des entraves de l'âme et inhibent le processus créateur. On rejette ascétiquement les tableaux à sujet, on cherche à créer par la contrainte, à partir de soi, des formes et les objets adaptés à ces formes, afin que le tableau ne soit qu'une émanation de l'âme la plus pure; christianisation de l'optique, le religieux libéré s'empare de l'art. Le visuel devient un contenu interne; à l'aune de l'expérience visuelle commune, on active un paradoxe d'immanence optique. Motif exclusif : le vécu du peintre, détourné des choses. Un lyrisme; on restitue sa propre oscillation intérieure. Dans ses écrits, Kandinsky identifie fréquemment la forme colorée et l'image sonore. En se concentrant sur l'image, on décharge la tension accumulée dans le processus psychique, on transforme celle-ci en juxtaposition dynamique, créée à partir de rapports de couleurs qui ne renvoient plus guère à une réalité extérieure. On se replie sur un centre psychique d'où irradient des formes psychiques, et on écarte le souvenir de l'extérieur au profit d'une limitation égocentrique. Dictature d'un vécu et d'une vision coupés des choses. Celles-ci entravent l'élan intérieur de l'individu, elles nous figent dans l'inertie conservatrice de la forme de l'objet, que l'on refuse de regarder parce que la chose pourrait menacer la sensibilité et contraindre à un détour. On cherche une expression directe du moi nouveau; au lieu de représenter des choses données, on cherche avidement à créer des formes annonciatrices de nouveauté. $\mathrm{Au}$ début, on est saisi par une folle hallucination, qui est une souffrance; les couleurs entraînent on ne sait où; on est encore sans volonté, jouet d'une contrainte - psychographique, et même impressionniste. Plus la sphère de représentation se restreint, plus l'ivresse est complète et brutale. On se défend contre elle en élevant le degré de conscience; les doctrines programmatiques, en effet, ne sont pas toujours au premier chef le produit d'un intellectualisme. Elles ont souvent la fonction de protéger des extases délirantes et destructrices, elles sont une réaction face à un dangereux débordement et à une tension difficilement soutenable, qui doivent être maîtrisés sous peine de mettre la vie en péril. L'idéologie est ici le produit d'une commotion brutale et elle traduit toujours l'origine fantasmatique ou hallucinatoire qui est la sienne en exposant moins de purs théorèmes que les généralisations d'un vécu subjectif, des impressions spirituelles.

On fuit les choses et les expériences optiques extérieures et on croit que la vision intérieure peut être entièrement coupée de l'extérieur; le retour au moi a pour prix la perte du monde vu. Le réel est stratifié en différentes catégories de valeur; c'est l'élan psychique qui est reconnu comme la plus significative, car il peut se nourrir de lui-même sans recours à l'extérieur; monologue circulaire. Le monde est là, pense-t-on - pourquoi le reproduire et entrer ainsi dans une vaine compétition avec Dieu, dont on ne sera que le copiste maladroit, incapable de rendre justice à la beauté de sa création. Tout au plus reconnaît-on une création artistique formelle, dont les formes s'assemblent en échelles d'objets; dans cette résignation devant la perfection de la création divine, on peut reconnaitre une attitude religieuse. On abstrait ainsi une intériorité ou un moi qui va, à un niveau transcendant, opérer des séparations, et qui devient dès lors, sur un mode fichtéen, le porteur des éléments. Réflexe exacerbé et tardif, peut-être, d'individualisme nietzschéen. Les formes se constituent et émergent à partir de l'intérieur; elles sont marquées par une nécessité psychique et justifiées par celle-ci. 


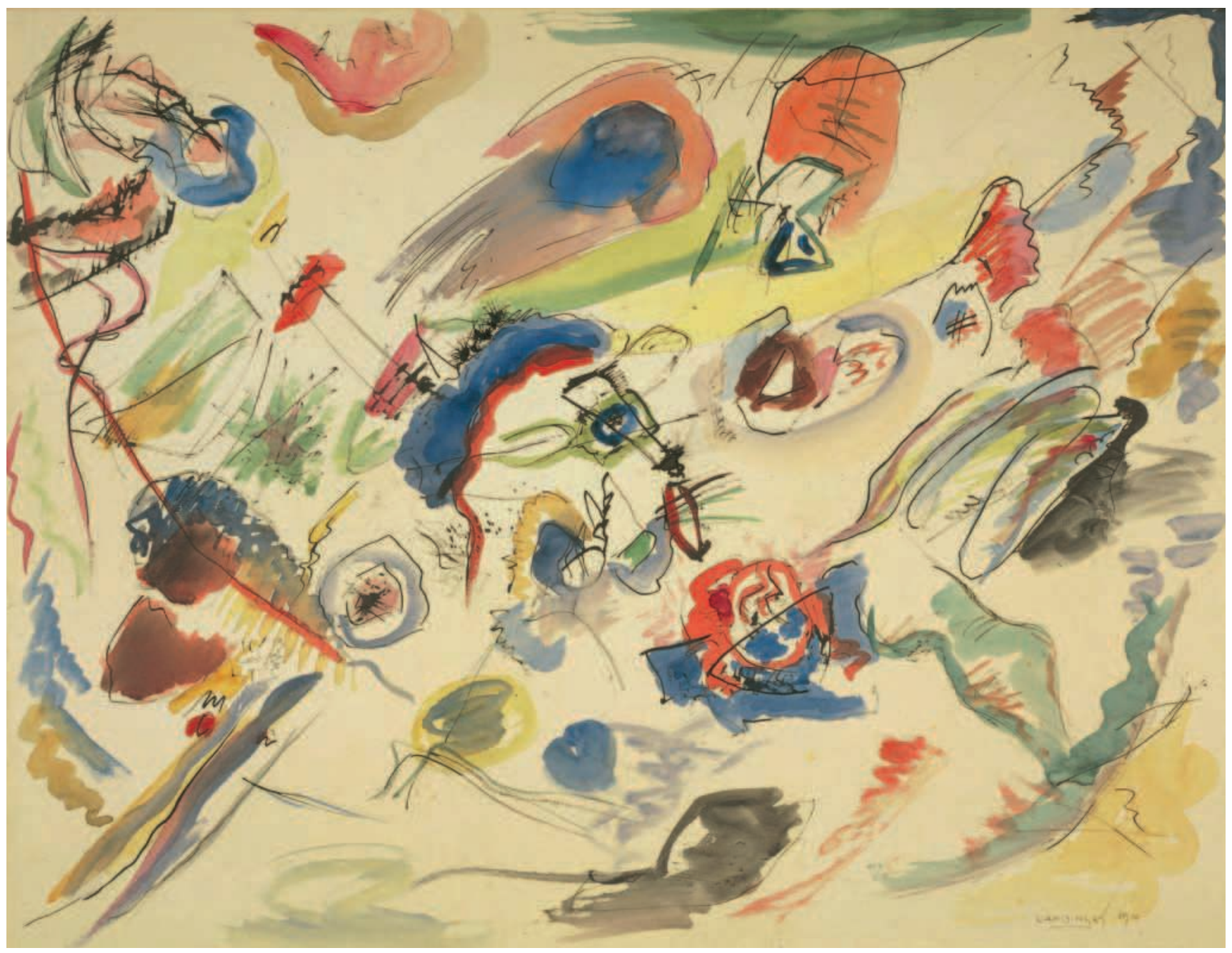

Fig. 10 Vassily Kandinsky, Aquarelle, 1910, collection Centre Pompidou, dist. RMN / Jacques Faujour @ ADAGP, Paris 2011. 


\section{TEXTES DE CARL EINSTEIN}

Chez le Kandinsky plus tardif, plus constructiviste, on pense peut-être aux formes géométriques qui résultent de la construction mathématique. Le style antérieur, fluide, a fait place à une pédanterie de la combinaison, et la contrainte dynamique se réduit désormais à un décor statique, pensé. On croit que les images vont acquérir une validité supérieure si elles contiennent des formes en apparence plus universelles. L'image doit ainsi recouper complètement le théorème qui généralise. Conséquence logique de l'erreur fondamentale de l'esthétique nouvelle, qui identifie ni plus ni moins les formes à des concepts. Pourtant, l'art se distingue de la science en ce qu'il revendique la possibilité de réaliser

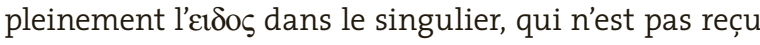
comme un universel mais comme un objet représenté concrètement et par là représentable.

À la place de l'image qui reproduit, on pose, pour compenser la résignation devant la perfection de la Création, un autisme, et l'on évalue désormais la réalité en fonction de sa proximité avec le moi; du point de vue psychique, son importance est d'autant plus grande qu'elle possède une plus grande proximité avec le moi. Le moment du souvenir, qui résiderait dans la mise en forme de l'objet, est déjà tenu pour impur - pourquoi ce détour alors que la peinture n'est que l'expression d'une intériorité ; il n'est pas nécessaire d'ordonner celle-ci par rapport au monde ordinaire; car il existe des domaines plus immédiats d'un point de vue spirituel. À l'aide de moyens d'expression lyriques et colorés, il s'agit de peindre des objets qui correspondent entièrement au sujet; il s'agit de refléter dans la couleur le contenu purement psychique; l'imagination dicte, et le monde donné est écarté parce que sa réfraction métaphorique affaiblit l'intériorité.

Kandinsky distinguait l'objet contingent qui rencontre fatalement le regard et la forme d'expression intérieure qui advient dans un état d'isolement subjectif. À cet élargissement du lyrisme subjectif correspond un rétrécissement de la sphère des choses; une sensibilité se manifeste qui rejette la transformation du donné comme un acte contraire à la spiritualité et à la créativité et trace un parallèle entre la peinture et la musique absolue; cette affinité renvoie à une dynamisation de l'image. Le sujet se crée l'objet formel qui lui correspond, et la création est étendue jusqu'à l'invention de l'objet lui-même; toute tautologie serait évitée si l'on ne se dessinait pas soi-même de façon d'autant plus désinhibée; un réalisme psychologique. On cherche passionnément à rendre manifestes, à partir de soi-même, les moyens de l'événement psychique, le sujet; on aspire à un déplacement des valeurs du réel; une humanisation égocentrique de l'image. On postule l'existence d'un dualisme des processus intérieurs et du monde extérieur, qui revêt une tournure presque chrétienne; on décrète l'union de l'esprit et de la sensibilité spiritualisée, tandis que les choses du monde visible sont rejetées comme des signes contraires à l'esprit, qui ne sont pas immédiats parce qu'ils ne sont pas le fruit de l'invention intérieure. Les processus qui ont une valeur positive sont ceux qui mettent en œuvre une spiritualité immédiate et n'ont pas subi l'affaiblissement métaphorique des choses et des anecdotes du reporter. Peut-être ne voit-on pas ici que l'image, justement, en tant que part du réel, domine et transforme désormais aussi ce réel.

Kandinsky croit que le motif détourne des éléments de la peinture. On se rappelle ici les Voyelles de Rimbaud et les multiplications de mots d'August Stramm, dont les poèmes naissaient d'un petit nombre d'éléments verbaux, par dérivations grammaticales et sonores. La peinture : un moyen de s'isoler spirituellement contre le monde. Si la peinture est un domaine autonome, elle possède nécessairement en elle-même des éléments autosuffisants. On absolutise donc et ignore l'aspect fragmentaire de toute pratique artistique, qui est limitée à une signification unique par la concentration. Une expérience immédiate de la couleur se coupe du donné; à partir de la libre perception des rapports de couleurs, on crée un objet coloré visionnaire. On se rappelle, de loin, les associations de couleurs de Delacroix et des impressionnistes; on avait cru identifier dans le mouvement de la couleur l'instance décisive de mise en forme de l'objet. En défendant de telles vues, Kandinsky atteste son appartenance à la génération de Matisse.

Kandinsky crée ses corps colorés en vertu d'une contrainte intérieure, d'une émotion qui se renverse souvent en interprétation symbolique, autrement dit littéraire. L'aliénation de l'objet contraint à la constitution d'une loi un peu indéfinie et souvent arbitraire; Kandinsky invoque la nécessité intérieure : "L'harmonie de la couleur se fonde sur le principe selon lequel l'âme humaine doit être affectée conformément à une fin. " C'est justement la dimension extraordinairement égocentrique de sa création qui l'a sans doute conduit à des généralisations et à la formulation de lois; on abandonne les motifs dictés par un objet et on veut atteindre une validité des contenus formels immédiats par la conformité du processus de création et de son résultat à certaines lois. On fonde l'intelligibilité de ces contenus formels sur le lien qu'on établit entre des rapports de 


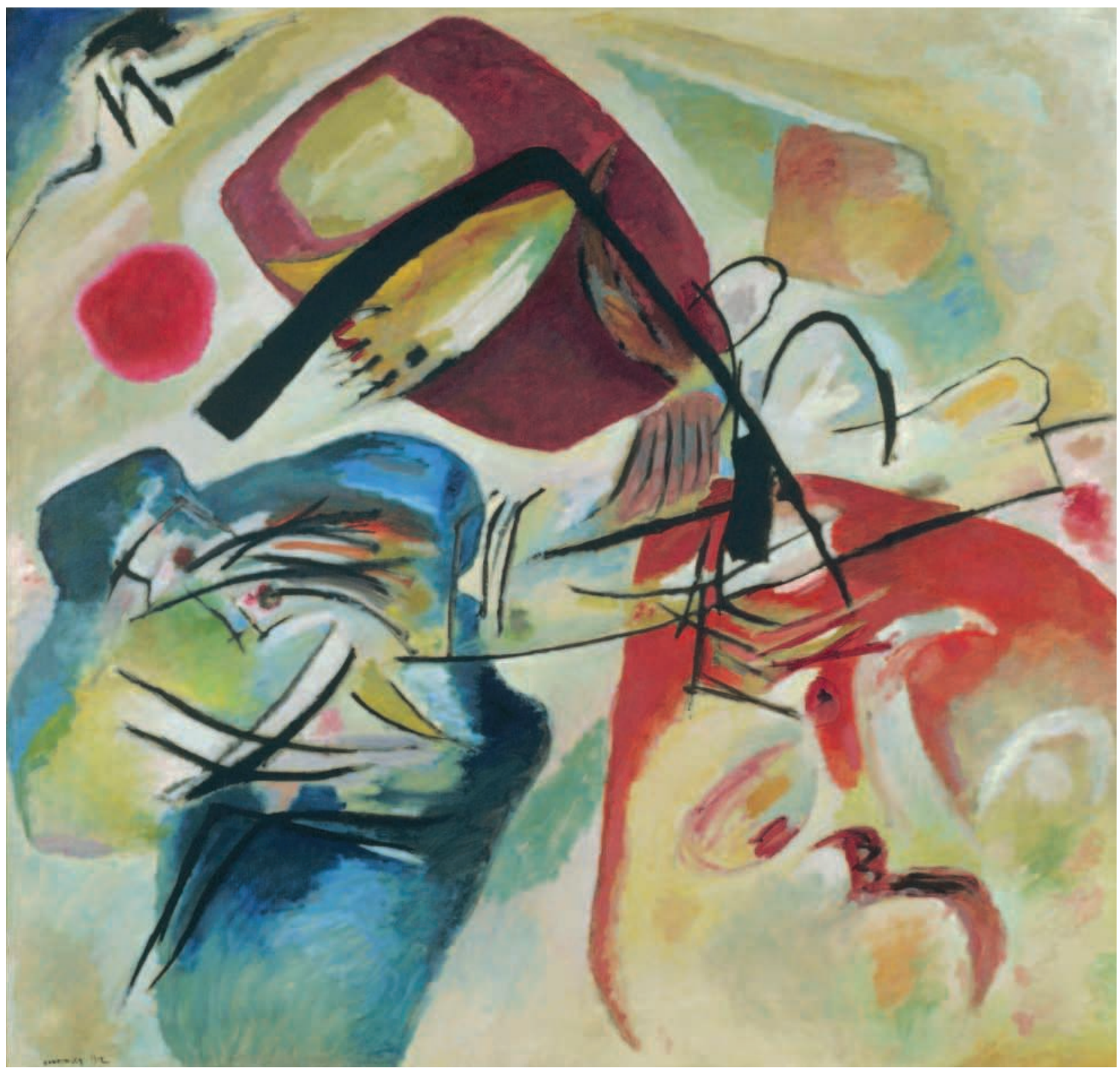

Fig. 11 Vassily Kandinsky, Avec l'arc noir, 1912, collection Centre Pompidou, dist. RMN / Philippe Migeat @ ADAGP, Paris 2011. 


\section{TEXTES DE CARL EINSTEIN}

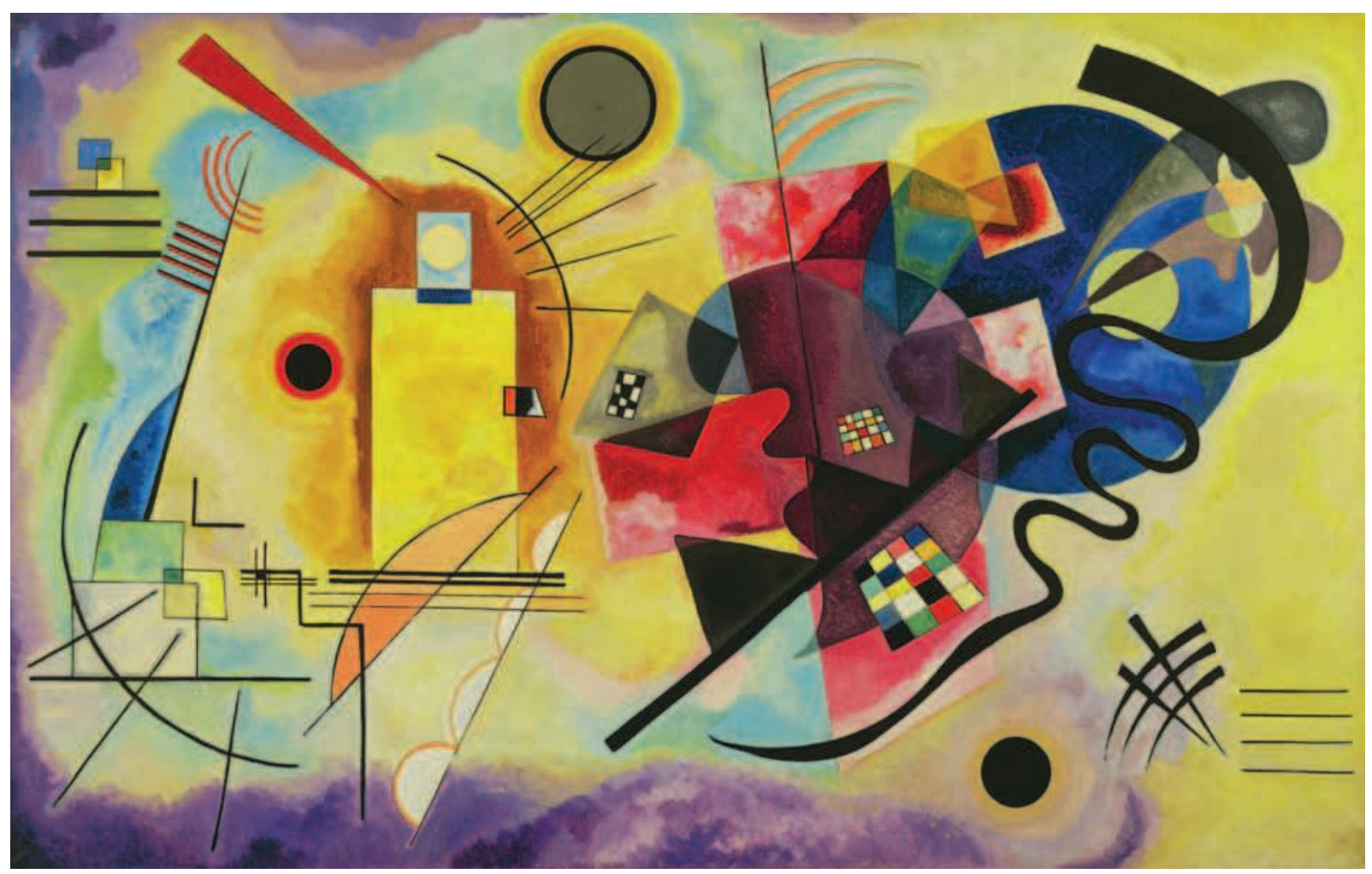

Fig. 12 Vassily Kandinsky, Jaune Rouge Bleu, 1925, collection Centre Pompidou, dist. RMN / Adam Rzepka @ ADAGP, Paris 2011.

couleurs et des associations musicales ou faisant intervenir d'autres sens, sans reconnaître ce que la constitution de lois psychologiques peut avoir de problématique; chaque loi psychique que l'on tente de poser, en effet, peut se renverser en son contraire, et nous n'avons guère les moyens de définir de façon univoque des lois des processus intérieurs. On confond ici, à n'en pas douter, causalité universelle et nécessité particulière, et la théorie perd ainsi son caractère contraignant; à la place, Kandinsky pose la «nécessité mystique» de la création, antérieure à toute théorie.

Severini a montré les faiblesses de son théorème mathématique, en observant que la construction quantitative ne permettait pas de cerner les qualités psychiques incommensurables qui interviennent dans la création d'une image. Kandinsky se plonge tout entier dans les sphères de l'âme prétendument vides de tout objet, et il les investit d'une charge symbolique pour aboutir à une doctrine de l'extase par la couleur. Celui que son subjectivisme isole est en quête de conformité à des lois intérieures, afin que ses actes ne s'abîment pas dans la solitude de l'arbitraire et qu'une loi contraigne le spectateur à revivre l'extase de la couleur.
La doctrine de l'absence d'objet de Kandinsky me semble reposer sur une erreur fondamentale. Il confond manifestement objet et figuration. Reproduire un motif et créer une figure en adéquation avec des processus psychiques ne revient pas au même. Cette erreur a été rendue possible parce que le théorème de Kandinsky est fondé sur les méthodes de la métaphysique ancienne, des méthodes incapables de fournir une représentation et une interprétation des processus dynamiques, dans la mesure où ces derniers sont immédiatement découpés en concepts, comme si, du puzzle dialectique des concepts ainsi isolés, il était possible de déduire une image des processus psychiques.

Il a été funeste que les Allemands, en matière d'esthétique et d'art, aient forgé une opposition entre l'art abstrait et l'art de la représentation, au lieu de comprendre que ce qu'on appelle «l'art abstrait » représente tout aussi concrètement, et même peut-être plus précisément, que l'art dit imitatif. Une seconde erreur fondamentale de cette esthétique est d'avoir constamment eu recours aux notions de monde intérieur et de monde extérieur, qui sont des abstractions artificielles, au lieu de garder en permanence à l'esprit que ces deux termes 
ne possèdent qu'une valeur heuristique ou de classification provisoire et qu'il n'existe pas réellement, dans le psychisme, de distinction conforme à cette antithèse linguistique.

Lorsque le problème du monde intérieur est posé, il faut avant tout étudier les bases psychologiques d'un tel questionnement et d'un tel acte de séparation. Nous pouvons constater que ceux-ci ont une cause principale : l'angoisse profonde devant le monde extérieur. Une fuite devant les choses qui doit rendre possible un entraînement médiumnique, dans l'isolement. On se détache de la civilisation des choses qui s'est développée de façon hypertrophiée. Peut-être pouvons-nous admettre aussi qu'il y a là des processus de mort. Quand on parle de monde intérieur et de monde extérieur, cela veut dire uniquement que seule une énergie psychique limitée peut être concentrée alternativement dans des domaines bien définis, en sorte qu'il est difficile - en raison surtout de la différence des termes - de garder consciemment à l'esprit les stades intermédiaires de l'échange d'énergie, qui font le lien entre ces domaines.

Il est significatif que Kandinsky ne soit pas vraiment parvenu à créer des formes à partir de tels dualismes métaphysiques : la projection dans le monde figuré lui demeura interdite. C'est précisément là que réside la principale faiblesse de son art, et c'est là la raison pour laquelle ses tableaux, en dépit de toute leur audace, ne furent pas autre chose que des décorations. Kandinsky reste pris dans la zone esthétique et ne parvient guère à la création ultime, c'est-à-dire à la création d'objets qui s'intègrent peu à peu dans le monde ou auxquels le monde s'adapte lentement. Par là, il appartient ni plus ni moins au type artistique qui, par indifférence esthétique, ne cherche qu'insuffisamment à transformer l'être. Kandinsky en reste à des formulations abstraites et ne comprend guère la réalité concrète et singulière des processus psychiques. À la place, il abstrait des éléments, et confond ainsi les découpages conceptuels et les processus psychiques mêmes. Il cherche à rattacher ces éléments abstraits aux processus psychiques par le biais d'une symbolique vague et à retrouver ainsi le flot des événements de l'âme. Mais ces éléments restent privés de vie et ne disent pas grand-chose de concret sur de tels processus. Nous sommes confrontés ici à l'erreur fondamentale d'une psychologie dépassée, qui entendait appliquer au psychisme des types d'abstractions issues de la physiologie ou des sciences naturelles.

À n'en pas douter, on fait appel à la raison pour se défendre contre l'irruption des extases; l'extase, inentamée par la multiplicité des formes, se laissera faci- lement rationaliser. Mais l'homme va tenter de lui imposer des contenus, afin de maîtriser l'existence par la métamorphose. L'expérience est multiple et n'est, par suite, guère ordonnée dans une unité formelle; l'extase tourne autour d'un point fixe, voilà pourquoi elle peut facilement être systématisée par l'entendement; nous connaissons de toutes parts la dialectique stérile du néant visionnaire; mais la part de création réside ici dans la variation des contenus. L'accent mis sur le sujet est un processus centripète, qui appelle une projection vers l'extérieur.

Le peintre exprime le contenu spirituel, psychique, par la couleur. C'est ainsi que Kandinsky aboutit à une sorte de caractérologie des éléments de couleur; il tente d'établir un canon; on veut se justifier ou combattre le sentiment de vertige suscité par des visions audacieuses. Dans un premier temps, Kandinsky tente d'expliquer le caractère des couleurs fondamentales, jaune, bleu, vert, rouge, etc.; il a principalement recours à des interprétations poétiques du contenu pour forger une sorte d'éthique des couleurs à partir d'une base générale émotionnelle et de choix littéraires personnels. On voit ici que l'objectalité est refoulée par l'interprétation psychique. Pourtant, la lecture poétique du caractère de différentes couleurs ne mène pas loin; la couleur isolée est marchandise. Kandinsky se préoccupe trop peu des liens d'ensemble entre les couleurs et ne voit pas les relations à partir desquelles elles reçoivent un sens précis. La forme est l'unification des relations de la couleur, de la ligne et du volume dans chaque partie du tableau et par rapport à chaque partie du tableau, qui doit être suscitée et rendue possible dans chacun de ses points. L'importance de l'image classique tient à cette multiplicité de variations sur ces relations, qui ont formé peu à peu une tradition. Kandinsky s'intéresse trop aux matériaux du marchand de couleurs, dont il livre une idéalisation littéraire; la couleur en soi n'est rien; la couleur n'acquiert une importance artistique que lorsqu'elle est modifiée par une forme et rapportée à une conception de l'espace, parce qu'elle est alors subordonnée à une unité formelle plus globale et que, dans ce cas, la tâche principale de la plastique, à savoir la création de formes, n'est pas éludée mais passionnément poursuivie.

La théorie de la couleur de Kandinsky est une poésie du tube de couleur; pas beaucoup plus. Si la couleur en soi possédait à elle seule un caractère aussi précis, le peintre n'aurait plus grand-chose à inventer et plus guère de variantes à introduire; dans le meilleur des cas, ce serait un arrangeur, qui maîtriserait moins 


\section{TEXTES DE CARL EINSTEIN}

l'outil de couleur qu'il ne serait maîtrisé par lui : il contiendrait alors déjà en lui les présupposés de la forme et les limites des possibilités d'invention. Cette approche théorique de la couleur était le résultat d'une expérience personnelle; à ses débuts, dans son travail, Kandinsky avait subi l'emprise de la couleur à un degré proprement hallucinatoire; il l'interprétait davantage sur un mode associatif qu'il ne la subordonnait à une représentation de la forme définie par avance; alors que la possibilité d'avoir recours à la couleur et à la ligne tient à la multiplicité de leurs mises en forme possibles, entre lesquelles l'artiste opère à chaque fois un choix. C'est justement cet abandon passif à la couleur qui a sans doute empêché Kandinsky de trouver accès à la création d'objets; sa doctrine restreint trop les possibilités de figuration, et c'est ainsi que, au lieu d'aller vers les choses imagées, il fit de la couleur, dont il se contenta dans un premier temps, un moteur et une finalité de l'image. Kandinsky exige la reconnaissance de son interprétation poétique, il en appelle à un spectateur "qui laisse le tableau agir sur lui directement". Soit on reprend alors l'interprétation subjective, élevée au rang de loi par une intuition mystique, et on se soumet à ce qui est présenté comme une nécessité interne, soit on ne voit là qu'un arrangement de plus ou moins bon goût.

Dans son traité Du spirituel dans l'art, Kandinsky a davantage donné dans la poésie associative sur le thème du décoratif qu'il n'a pris position au sujet de la forme complexe. Il livre des figures colorées sur une surface; dans cette pure surface, qui ne cherche jamais à intégrer des volumes, on assiste peut-être déjà à un affaiblissement de la chose dans sa plasticité; comme on ne part que de la couleur musicale, on ne voit plus aucun volume; l'appréhension tectonique de l'espace entravait, à n'en pas douter, la succession des sensations de couleur. On craint peut-être de banaliser son intériorité en laissant l'expérience de la forme déboucher sur des objets; on identifie trop l'objet de l'image à des choses conventionnelles et on ne voit pas que l'objet se trouve à la périphérie de l'image, là où créateur et spectateur ne font plus qu'un, en sorte que celui qui regarde est possédé par de nouveaux signes de forme et contraint de modifier son image du monde. Kandinsky remplace la forme par le commentaire coloré d'éléments abstraits; par là, ses tableaux se réduisent de plus en plus à une chaîne associative d'illustrations d'un théorème insuffisant.

Chez Kandinsky, le spectateur du décor coloré découvre un ensemble d'intuitions spirituelles qui ne sont guère objectivables, et la voie est ouverte à une poésie indéfinie. Dans l'image d'un objet, en revanche, le profane est affecté par une figure précise et il l'est en même temps d'un point de vue formel. Plus tard, Kandinsky fit usage de formes géométriques; en Russie, il fut influencé par des courants constructivistes.

Le sens de toutes les images est l'influence qu'elles exercent sur le monde qui nous est imposé; par là, celuici est davantage humanisé, et il importe, sous tous les rapports, d'arracher l'art à la limitation esthétique. Kandinsky en reste à des éléments groupés de façon charmante; mais il ne voit pas que les images ne prennent sens qu'en devenant les signaux et les indices de notre création d'un espace et en nous permettant d'élargir le monde. Par là, des besoins biologiques sont satisfaits, et on s'affirme contre la création imposée. Kandinsky, lui, s'enferme dans une sorte d'angoisse de l'objet, tendrement résignée, qu'il renforce en se fondant sur la vieille construction d'une antithèse entre l'esprit et l'objectalité du monde, sans même signaler, ne serait-ce qu'en passant, ce que la résistance objectale peut avoir de fructueux (un de ses avantages les plus fréquents). La liberté d'expression à laquelle il aspire est fondée sur une négation limitative; son purisme spirituel recèle encore une part de vieille métaphysique chrétienne: la doctrine de l'esprit absolu, hermétique. De ce point de vue, il se rapproche des suprématistes, par exemple. Soit le spectateur accorde foi à ce dogme, soit il voit dans les travaux de Kandinsky des décorations sans audace excessive. Kandinsky est entièrement dépendant de l'humeur du spectateur, des contenus dont celui-ci emplit la décoration qui lui est présentée, et on peut se demander si les associations du spectateur peuvent recouper celles du peintre. L'absence d'objet permet au spectateur de se faire poète et de créer, et ces œuvres finissent par devenir l'illustration d'un contenu plus ou moins littéraire. Kandinsky n'a pas compris la distinction entre l'objet et la figure livrée dans l'image; cette dernière limite l'arbitraire interprétatif du spectateur et arrache l'image à l'isolement esthétique. Il n'en irait pas de même si Kandinsky parlait de la décoration en architecture. Mais lorsqu'il s'agit de l'image autonome, la tautologie des couleurs n'est guère suffisante.

Kandinsky a ignoré des forces extrêmement importantes, notamment la mise en forme de l'espace; sa théorie est trop pauvre, et elle n'est rien d'autre que la généralisation d'une expérience enfermée dans le moi, qui, dans sa simplicité et ses émois par trop personnels, s'est renversée en justification esthétique pour trouver un apaisement. 
La liberté recherchée a été perdue au profit d'une orthodoxie littéraire, et ce mince vécu a dû faire l'objet d'une justification didactique; par crainte peut-être d'un volontarisme excessif, on a tenté de se rattacher aux Anciens par la théorie; c'est à cette source que Kandinsky a puisé le peu qui s'accordait à ses vues. L'arrachement à l'objectalité est un stade intermédiaire vide avant la découverte de formes objectales nouvelles; enfermés dans ce monde trop étroit pour nous, nous devons, en effet, réinventer sans cesse.

Vérisme et colportage ont été une réaction à la liberté étroite et poétisante de Kandinsky. On s'est mis à condamner la subjectivité poétique; on ne voyait plus en elle qu'un obstacle entre l'image et l'objet à représenter. On voulait décrire méticuleusement. Le subjectif était interprété comme un vertige artistique né de la sentimentalité individuelle et on espérait trouver dans l'objet assez d'éléments pour livrer une représentation scrupuleusement exacte. Avec ces descriptions presque serviles, davantage dignes du journalisme, on était désormais à la botte de la nature et des objets. La subjectivité, l'extase poétique étaient dévaluées; on avait renié l'anecdotisme de l'abstrait, mais pour tomber aussitôt dans le reportage et le journalisme.

\section{Paul Klee}

De Marc et Kandinsky émanait une menace : la peinture comme retraite. Kandinsky ne parvint pas à constituer des formes ni, par suite, à métamorphoser la réalité ou à en forger une nouvelle. Son esthétique était plutôt de type négatif. Voilà pourquoi il se satisfit d'un outil de second ordre, l'ornement lyrique; on reste dans l'étroitesse monologique et mécanise ses visions de façon toujours plus irrémédiable. Kandinsky prit pour base une symbolique trop facile, et sa peinture finit par se confondre avec, disons, les allégories d'un théorème. On peint le fourre-tout facile de l'abstraction et une attitude ascétique aboutit, à la fin, à une suite de commentaires verbeux.

On a souvent rapproché Klee de Kandinsky, mais il me semble que l'un et l'autre poursuivent des objectifs tout à fait différents, sinon diamétralement opposés. Pour Klee, une chose paraît décisive : saisir, en même temps que l'image, les figures dont elle trace le contour. Sa vision pénètre des couches plus lointaines et plus décisives que celle de Kandinsky; lui en reste aux prémisses.

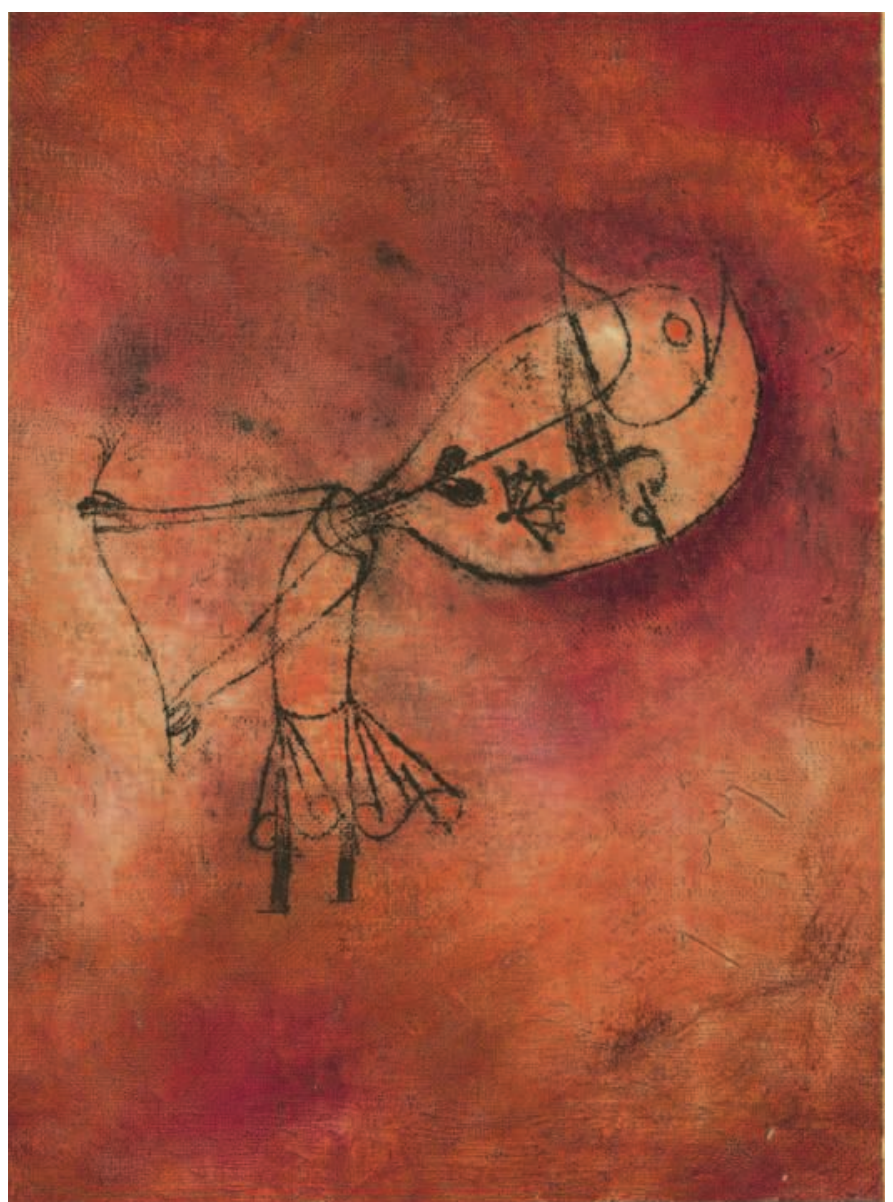

Fig. 13 Paul Klee, Danse de l'Enfant pleurant II, 1922, Munich, Pinakothek der Moderne, collection Moderne Kunst ๑ BPK, Berlin, dist. RMN / image BStGS.

Il est bien rare que l'homme se transforme et transforme le monde qui l'entoure; à l'aune de tout ce qui a été hérité et conservé, la nouveauté ne brille que très faiblement. La fin du xix ${ }^{\text {e }}$ siècle a été une époque de description; la métaphysique qui avait mis en question les choses et leur existence a fait place, disons, à une métaphysique des corps solides; la perception passait désormais pour univoque et elle était tenue pour le fondement unique des expériences vécues. Le mythe était mort, il n'existait rien au-delà des choses, ou seulement de vagues conjectures, les processus psychiques n'étaient tout au plus qu'une source d'erreurs et on pouvait sans crainte s'abstenir d'en tenir compte.

Une fois qu'on eut éprouvé et constaté que l'espace, par exemple, pouvait être une création absolument libre, on en vint à se demander, puisque l'espace pouvait être forgé par l'homme, s'il était également possible de créer des formes nouvelles. La question d'une possibilité du mythe était posée. Une telle création, au 


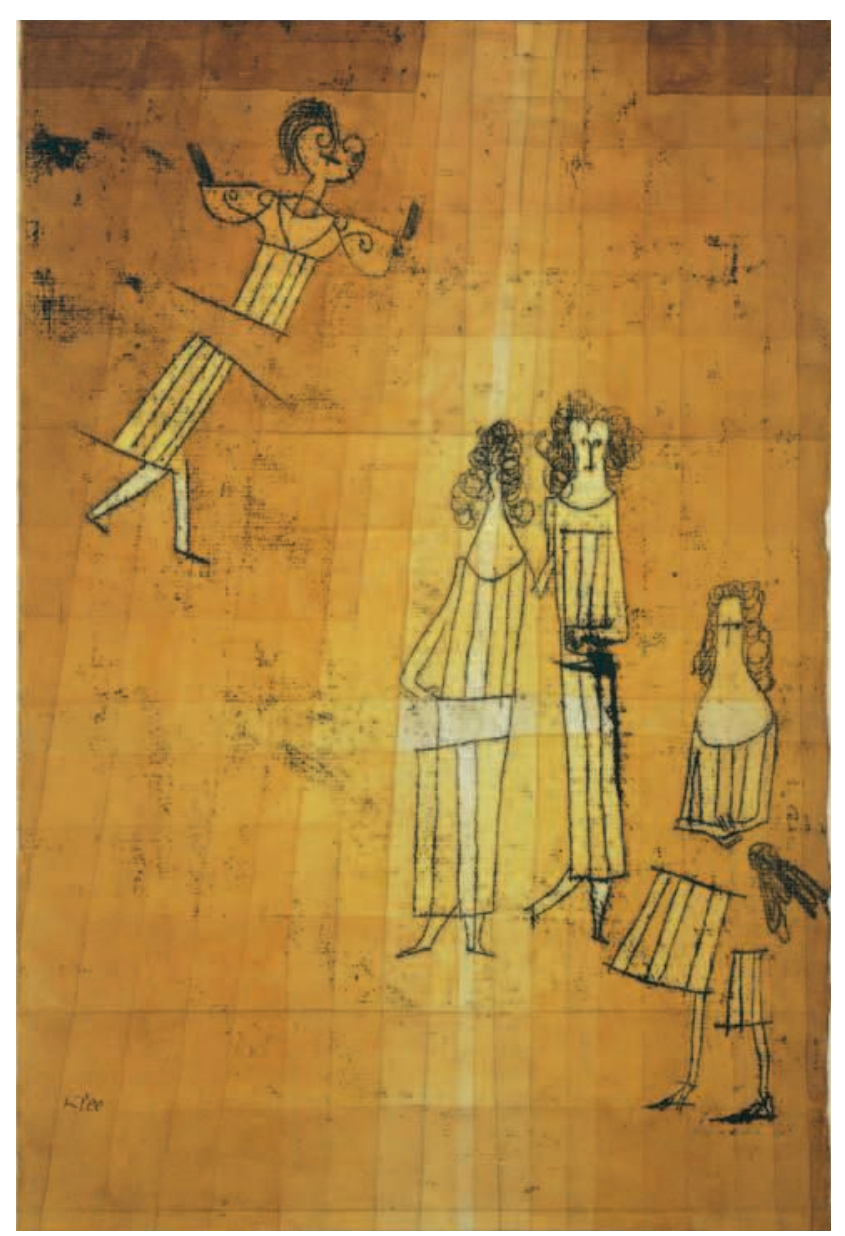

Fig. 14 Paul Klee, Scène entre filles,1923, Berlin, Nationalgalerie, Museum Berggruen (SMB) ๑ BPK, Berlin, dist. RMN / Jens Ziehe.

demeurant, est absolument aux antipodes des légendes anciennes. Elle est engendrée dans l'isolement et se développe en rupture avec son milieu, alors que, par le passé, le mythe était l'expression du collectif. Le mythe nouveau doit donc être entendu comme révolte, alors que, auparavant, il préservait le vécu et l'héritage communs.

L'homme ne cesse de transgresser le réel, s'il veut vivre et s'affirmer. Pour s'exprimer, il ne se contente pas de l'existence attribuée par le destin et des formes qui lui sont liées; il a l'impulsion et le don d'accomplir des actions qui dépassent tout cadre préétabli. Dans l'inconscient, nous possédons des forces qui, longtemps, n'ont guère été utilisées et n'ont pas été adaptées à la vie collective; dans le cas contraire, en effet, nous n'aurions pas été contraints de les refouler. D'un autre côté, le même inconscient où sommeillent les forces non encore adaptées stocke les héritages oubliés par le conscient, y compris les atavismes et leurs effets automatiques. Ces strates de type contradictoire, à la fois sur le plan psychique et sur le plan de l'histoire, se manifestent de façon caractéristique dans toutes les tentatives pour forger de nouveaux objets ou de nouveaux mythes. D'un côté, une discordance se fait sentir par rapport au monde environnant et, de l'autre, on observe une étrange conformité à des situations et des structures anciennes; autrement dit, la formation de nouveaux objets est souvent imprégnée d'archaïsmes.

Il est une phase de la vie, l'enfance, où nous avons le courage d'inventer des objets, car nous n'avons pas encore alors à prouver la validité de notre réflexion en accomplissant des actes aux ramifications étendues. Celui qui juge superficiellement croit que l'enfant joue, alors qu'il fait des sauts singulièrement alogiques que l'adulte ne comprend guère. En fait, l'enfant projette dans le présent quelque chose de nouveau, qui est encore inconscient, mais, en même temps, des atavismes hérités, que l'adulte oubliera peut-être parce qu'il ne les mobilise que rarement dans l'action pratique. On pense alors, tout à fait banalement, que l'enfant ment ou qu'il est mythomane. Puis on devient adulte et on oublie ces deux forces, parce qu'on ne peut plus s'autoriser, alors, des actions qui ne soient pas en accord avec le milieu et avec certaines finalités pratiques. Les forces mythiques de l'enfant sont ainsi oubliées.

Ce sont justement ces forces qui exercent leur emprise sur l'artiste qui tente de forger des objets de façon indépendante. C'est ainsi que nous interprétons, au premier abord, le caractère parfois infantile ou primitif de la formation de nouveaux objets. Toute nouveauté contient en même temps une régression, car elle provoque la réaction de couches longtemps inutilisées. Un art tectonique nouveau éveillera par exemple les souvenirs refoulés de courants tectoniques anciens: l'art sumérien, l'art grec archaïque, l'art byzantin se rappellent à nous à la faveur du présent. Au demeurant, l'art actuel est ainsi historisé. Un courant psychographique éveillera des réminiscences ornementales, en évoquant, par exemple, l'art néolithique, l'art nordique ancien, etc. Car l'histoire est formée à partir du présent; la sélection du passé s'effectue en fonction de la signification actuelle qui lui est attribuée. Ce fait explique le changement continuel des images historiques; en même temps, la possibilité d'une écriture objective de l'histoire se trouve par là même contredite. C'est ce qui expliquerait le rapprochement entre l'art actuel et le primitif. Cependant, l'archaïsme psychique est plus significatif, d'un point de vue humain, que le seul archaïsme formel. 
On a souvent voulu souligner une parenté avec les dessins des schizophrènes. Ceux-ci s'emploient à affirmer, avec succès, une indépendance très nette vis-à-vis du monde qui les entoure; leurs représentations et leurs signes sont souvent fixés à un stade infantile. Pour eux, ces signes sont à ce point vivants qu'ils deviennent des êtres et des objets. Nul doute qu'il existe une certaine parenté entre le schizophrène non adapté et l'artiste qui invente librement. L'un et l'autre, pourtant, se distinguent rigoureusement sur un point : de façon générale, le schizophrène est attaché à un petit nombre de types, il est possédé sans retour et ne peut guère changer son monde de formes; ses représentations tournent autour d'un certain nombre d'idées fixes; des pans entiers de sa conscience sont peut-être susceptibles de fonctionner, mais, en raison de cette fixation, il n'est pas capable de les utiliser avec succès et d'abréagir. L'artiste, quant à lui, opère une abréaction suffisante et un transfert de son état sur le spectateur, et parvient ainsi à une autoguérison; il est dès lors en mesure de former sans cesse de nouveaux signes, avec une rapidité remarquable qui est la preuve de sa vitalité renforcée; tandis que le malade, lui, réussit dans le meilleur des cas à oublier son idée fixe pour se protéger d'elle; le plus souvent, il paie ce dernier refoulement d'un déclin de sa personne. Pourtant, nous ne regardons pas les fous comme de simples malades; nous voyons bien plutôt en eux des hommes qui n'ont pas la capacité, ou la volonté, de se soumettre aux conventions communes, et donc des êtres qui possèdent, dans une mesure inhabituelle, des forces particulières et un esprit singulier. Cependant, chez les schizophrènes, la masse des archaïsmes infantiles semble le plus souvent empêcher la formation du nouveau, alors que, chez l'artiste, l'accent majeur est mis sur l'innovation.

Klee a apporté à l'art allemand un tournant décisif. Avec lui, on est enfin sorti de la phase négative de destruction de l'objet. Il a apparemment été nécessaire, pour en arriver là, de passer par la zone neutre de la création ornementale, indifférente à l'objet. Avec Klee, un problème humain crucial a été formulé : celui de la métamorphose et de la reformation du monde par l'homme. L'homme a ainsi reconquis la force magique que, depuis longtemps, il n'osait plus utiliser. Le primitif, comme l'enfant, croit créer et définir des êtres et des choses par le biais des mots, des récits et des images. Là aussi, par conséquent, nous rencontrons dans ces formations nouvelles des cas typiques de régression.

Le réalisme se charge alors d'un sens plus profond : ce n'est plus une création qui imite la réalité ou la reproduit, mais la création nouvelle d'une réalité concrète. Cette transformation plastique fait nécessairement appel, nous semble-t-il, à trois forces : 1 . La retranscription médiumnique, c'est-à-dire l'abandon sans inhibition à des processus psychiques non encore adaptés, autrement dit une technique de transe; 2 . Les forces tectoniques, c'est-à-dire le contrôle et la prise de conscience des visions et l'intégration des expériences vécues de façon isolée par l'individu dans un ensemble de signes possédant une validité collective; 3 . L'identification avec une figure nouvelle, autrement dit la force de métamorphose.

En d'autres termes : une image parvient à constituer une totalité psychique quand elle contient différentes strates psychiques primaires. Ainsi, la question de l'image comme totalité ne se réduit pas, comme on le suppose en général, à un problème formel : la question qui se pose avant tout est celle de la totalité psychique. Le plus souvent, cependant, c'est une certaine strate psychique qui prédomine dans les images, car cellesci sont majoritairement des fragments qui ne rendent compte que d'un aspect unique, d'un point de vue psychique. Peut-être est-ce là la raison pour laquelle ils activent des courants particuliers dans les strates les moins abréagies dans l'image elle-même.

Pour Klee, il s'agit d'introduire dans la réalité des formes et des objets; en d'autres termes, le sens du mythe n'est plus la préservation du passé, mais la révolte contre l'existant. Lorsque, dans l'image, des formes nouvellement inventées rencontrent des formes héritées du passé, une tension grotesque s'instaure, aussi bien d'un point de vue psychique que d'un point de vue formel, et deux registres différents de réalité peuvent alors se persifler l'un l'autre.

L'une des fonctions décisives des images semble être de nous libérer des standards corporels imposés. Combien ce besoin est profondément ancré en nous, c'est ce que prouvent les tendances instinctives que l'on désigne sous le nom de perversions érotiques. Elles signifient que l'on ne se contente plus de la réalité corporelle physiologiquement donnée; l'imagination serait ainsi une forme sublimée de perversion ou, ce qui est plus important, une réaction psychique contre la fixation érotique.

Kandinsky croyait qu'il suffisait de rejeter ce monde sur un mode ascétique et de dessiner, à la place, des ornements poétiques; on adopte alors une attitude d'indifférence artistique à l'égard de la forme. Ce point de vue correspond en gros à la transe sans objet du mystique, qui s'efforce de s'affranchir des 


\section{TEXTES DE CARL EINSTEIN}

représentations et des impressions pour atteindre le néant et le repos sans inquiétude.

Chez Klee, un tel parti pris est une phase destinée à purifier l'homme, afin qu'il devienne réceptif à des choses nouvelles et acquière le don de les accueillir. Chez Kandinsky, on peut parler d'une angoisse d'identification aux formes ou aux choses, quelles qu'elles soient; l'art comme tentative de fuite. Voilà pourquoi on détruit l'art et pourquoi fait défaut la force de la métamorphose.

Dans l'identification, on atteint un état primitif, enfantin; en l'occurrence, on reconnaît à la représentation une activité et une force presque aussi grandes que les siennes, comme l'enfant parle des choses et des jouets en les assimilant à des êtres et vit avec eux comme avec des êtres. Cette confusion se traduit, chez l'enfant, par le fait qu'il parle volontiers de lui-même à la troisième personne. On pourrait faire ici le constat d'un stade intermédiaire d'animisme qui disparaît peu à peu, étant donné que l'adulte distingue sur un mode de plus en plus conscient des êtres conscients et d'autres qui ne semblent pas disposer de cette force; en d'autres termes, une rationalisation de l'existence est accomplie, avec une nette survalorisation de la sélection rationnelle.

Un trait caractéristique de l'art de notre temps demeure le fait qu'un tournant anti-rationaliste a été opéré. On a ainsi trouvé le courage de peindre des formes et des images au premier abord absurdes en apparence, c'est-à-dire des images qui ne sont guère régies par un ordre formel « rationnel ». Il apparaît, au demeurant, que la raison est plutôt une entrave, une limitation psychique et une façade derrière laquelle des forces plus décisives, trop longtemps cachées, étaient en sommeil. On pourrait presque dire que la raison est un moyen de réagir contre les couches psychiques non adaptées et d'empêcher leur action.

La conception rationnelle de l'existence consiste dans une description univoque de celle-ci, qui peut s'appuyer sur des preuves. La raison nous cantonne aux expériences et aux formes dont nous sommes capables d'apporter une preuve visible, par exemple en comparant une nature morte avec les vrais fruits et les vrais vases; par là, elle limite la créativité.

Pourtant, nous sommes continuellement confrontés à des phénomènes élémentaires et indémontrables, comme le rêve et le miracle, dans lesquels l'âme agit apparemment sans se préoccuper de l'exactitude physique ni de la justesse formelle extérieure. La raison impose à l'homme une monotonie idiote de l'exis- tence et des formes que, dans le meilleur des cas, il fait varier et réordonne; une limitation fatale. Par le passé, on se défendait contre une telle monotonie des formes au moyen des mythes, la constitution des formes n'obéissait pas à des déterminations esthétiques, mais religieuses. Un symptôme social de ce désir de transformation des types peut être observé, chez les primitifs, avec l'exogamie, qui est conditionnée, en fin de compte, par un besoin de modification du type permanent. On veut se libérer de l'enfermement dans un standard planifié, afin d'acquérir des forces physiques et psychiques inconnues ou nouvelles. Un autre moyen d'y parvenir était la métamorphose en animaux, plantes, fleuves, etc. La même pulsion est à l'œuvre quand, aujourd'hui, on recherche de nouvelles configurations formelles; on rejoue le drame fondamental de la métamorphose, du changement de forme. Il est extrêmement facile de la mettre en scène dans l'image, puisque toute forme peut avoir une infinité de significations. Par suite, il faut insérer dans toute forme un certain nombre de partis pris d'objet; en d'autres termes, pour rendre les formes univoques, il faut les préciser en leur donnant un certain contexte d'objet. Par là, on touche à la question de la désignation de l'image, ou du titre. Les titres, chez Klee, font penser aux formules magiques d'autrefois. Ils contiennent peut-être la première suggestion qui s'exerce sur le spectateur et par là, son expérience de l'image se trouve dans un premier temps circonscrite.

Nous avons évoqué les trois forces plastiques fondamentales, l'écriture, les formes construites et la représentation de formes complexes.

Dans la retranscription passive s'exprime la capacité de souffrir, la force médiumnique; des formes à la volonté végétale vont ainsi apparaître. Dans le tectonique, nous tentons de nous défendre contre les divagations du rêve, de lui imposer des limites et un cadre qui lui font violence. Dans le psychogramme, on cherche à préserver la dynamique des processus en ne retranscrivant que les signes immédiatement nés de la stimulation; en d'autres termes, on n'impose pas à ces termes un ordre fixe et prédéterminé. À l'inverse, on entre dans la phase tectonique lorsqu'on entrave et contrôle les expériences vécues en les ordonnant dans des formes générales, durables, qui installent des constructions. L'artiste plasticien dispose ici de deux éléments que nous retrouvons aussi dans la langue. Au psychogramme dynamique correspondrait la forme verbale et, à l'immobilisation statique, la notion: le substantif. Il est caractéristique que le nomade en transit ait recours, en matière d'art, à des formes dynami- 


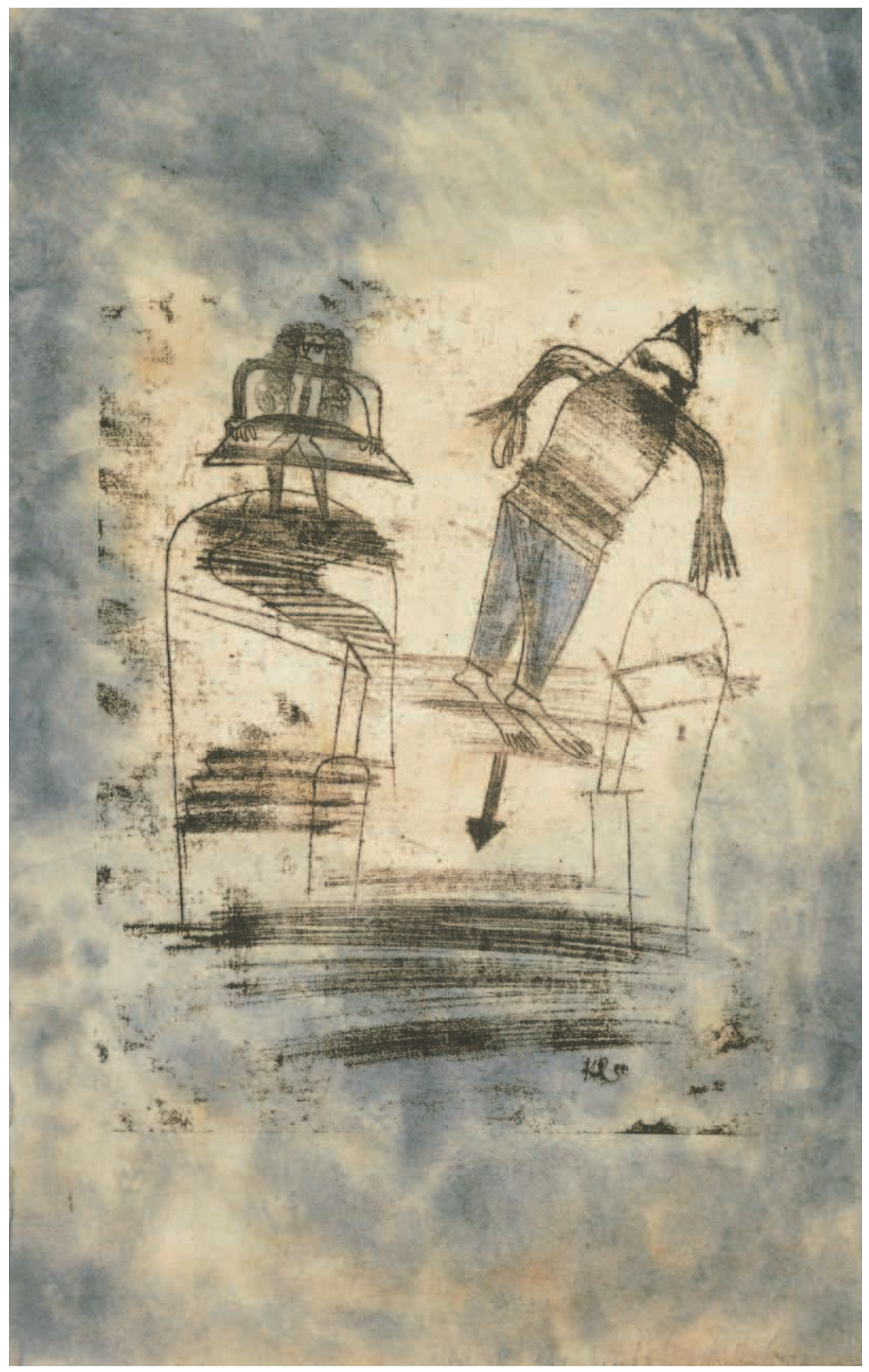

Fig. 15 Paul Klee, Manie de la persécution du Pierrot, 1924, collection Centre Pompidou, dist. RMN / Philippe Migeat. 


\section{TEXTES DE CARL EINSTEIN}

sées, c'est-à-dire tente avant tout de préserver le mouvement, tandis que le colon, le cultivateur, immobilise ses expériences vécues dans des formes tectoniques ou cristallines, conformément à sa propre sédentarité. À cette forme de vie conservatrice correspondaient le matriarcat, les domaines de la sédentarité, la maison.

Les domaines végétaux du calligramme montrent les hommes comme des plantes qui flottent au vent ou sont froissées par le sens du toucher, entièrement livrées aux éléments. Dans les formes apparemment géométrisantes, on cherche à mettre en place des figures plus résistantes, plus conscientes, en un sens, des êtres cristallins, dans lesquels on projette les pierres et l'immobilité. Par là, ces figures sont rapprochées de formes plus éloignées, leur sont rattachées d'emblée par leur caractère formel. L'homme-plante serait peut-être le rêveur inconscient, tandis que les figures tectoniques sont le produit d'une défense contre le rêve et un résultat de la volonté.

Ainsi se distinguent déjà, chez Klee, deux couches de l'âme antagonistes, produisant une disposition contrapunctique, très importante pour son art : un humour soumis à la tension de la contradiction, une sorte d'ironie romantique.

Par l'écriture médiumnique, on rêve de retourner dans le monde végétal du toucher; ou bien, par le tectonique, on accède aux lents domaines du minéral, où les formations se construisent à angles droits, de façon volontaire; ou bien un reflet des étoiles se mire dans un homme-étoile. Des formes mouvantes peuvent naître de l'air, de l'eau ou du feu et les signes des stimulations psychiques sont ainsi rattachés, de façon surprenante, aux anciens éléments. La forme ne serait dès lors qu'une synthèse de différentes forces cosmiques, l'âme et la personne le produit de leur tension et de leur différence. En d'autres termes : les formes rendent possibles d'extraordinaires rapprochements et métamorphoses de la figure en ce qui semble étranger à son être; et, dans la métamorphose, on observe que les phénomènes et les êtres se rattachent entre eux de manière fonctionnelle. La forme signifie désormais davantage qu'une stimulation esthétique: en l'occurrence, la force de l'autométamorphose et l'unité du processus métamorphique. D'un autre côté, cependant, les plantes et les pierres acquièrent désormais une puissance de métamorphose, elles se transforment en êtres différents et nouveaux, agissent en eux et acquièrent ainsi une force de vie identique à celle de l'homme. L'ordre hiérarchique positiviste établi à partir de la conscience est violemment ébranlé; par le biais des formes, on peut, en d'autres termes, créer un ordre de valeurs du monde ou le bouleverser. Par l'image écrite médiumnique, la forme se configure automatiquement comme un résultat final, ou bien comme un effet du hasard, la transcription est une quête de forme, tandis que, dans le tectonique, ce qui correspond à la figure est souvent un présupposé, obtenu par la contrainte de la volonté; on se fie alors d'emblée à un ordre établi.

Si la plante, le minéral, l'astre et l'homme sont quasiment mis sur le même plan, une plante humanisée peut entrer en action et se substituer à l'homme, ou bien un sentiment se transposer dans la structure d'un minéral. Une telle confusion des phénomènes est certainement enfantine; l'enfant accepte toutes choses comme des compagnons de jeu égaux en droits, dans une certaine mesure, et leur prête volontiers ses forces psychiques en les projetant en elles, parce qu'il n'est pas encore séparé d'elles par la fixation d'une conscience de soi. Voici qu'une branche pousse à la place d'un bras, ou qu'une tête devient une fleur. L'homme adulte, c'est-à-dire standardisé, désigne ces pulsions de métamorphose sous le nom de jeu. La caractéristique du jeu enfantin est que l'enfant n'est pas encore inféodé à des objectifs pratiques bien définis et n'est donc pas enfermé dans la sélection bornée des processus simplifiés par la logique. Il est ainsi capable d'agir et de vivre de façon discontinue, dans des analogies ou des configurations symboliques, alors que c'est seulement dans le rêve ou dans la poésie que l'adulte s'autorise des expériences qu'il peut difficilement intégrer dans le quotidien rationalisé, qu'il oublie ou devant lesquelles il est dépassé et plus désarmé que l'enfant. Nous pouvons dire que l'enfant accorde aux choses une réalité plus grande que l'adulte. Il est vrai que, de temps en temps, il se venge d'elles en les détruisant dans un accès de colère, d'une façon presque incompréhensible pour les adultes. Cela signifie que, par intermittence, les prémisses d'une conscience de soi et le besoin d'une supériorité de la volonté se manifestent; ou bien on adapte les choses au flot de l'émotion et l'enfant entre alors dans la phase de destruction des objets. L'instinct de jeu pousse là encore à ne pas accorder foi aux configurations données, mais à les métamorphoser de façon fabuleuse.

De même que l'enfant aime les poupées ou les marionnettes, nous rencontrons chez Klee des hommes-marionnettes, c'est-à-dire des personnages qui cachent leur volonté et leur jeu. Comme dans les rêves, nous voyons chez Klee des escaliers raides d'où on dégringole, des pièces où on vole ou, comme dans les 
cauchemars, des corridors étroits dont l'issue est obscure. Souvent, l'enfant détruit une poupée ou un jouet, mais il conserve soigneusement une partie de ces objets qui, pour lui, représente le tout, un bras de la poupée, une roue de la voiture, etc. À n'en pas douter, nous avons affaire ici, comme chez le primitif, à une composante devenue décisive pour l'art de notre temps : une partie représente le tout. Cela signifie qu'une stimulation partielle suffit à déclencher l'intégralité du processus psychique et que la représentation partielle d'une image est complétée par le psychisme. Ainsi, l'énergie psychique peut se ménager un espace plus vaste, puisque toutes les parties de la représentation n'ont pas besoin d'être fixées. Il apparaît que la figure n'a pas seulement pour fonction de représenter et d'agir comme un double, mais qu'elle peut produire, d'un point de vue psychique, l'effet d'un symbole ou d'un instrument magique. Il suffit de disposer des cheveux ou des ongles d'un homme pour acquérir un empire sur lui. On perce le nom avec une aiguille, du sang coule sur le papier et celui qui porte le nom meurt à distance. Tous ces processus magiques sont récurrents chez Klee et attestent une réaction profonde contre le rationalisme. Les images de Kandinsky sont démontrables et rationnelles. Les formes ne sont pas métamorphosées en complexes de figures de son invention, on reste dans la zone de l'indifférence artistique et les éléments ne sont pas travaillés pour produire une réalité efficiente. Mais ces figures peuvent s'introduire dans la réalité; il en résulte alors une opposition entre la vision et l'être, entre le rêve éveillé et la représentation commune. Résultat: l'humour des tableaux de Klee, une tension des contrastes.

Nous le voyons : l'humour est absolument opposé à toute caricature, qui naît quant à elle de la comparaison intellectuelle entre l'idéologie et la réalité. L'humour peut être l'indice d'une solitude, d'un éloignement de l'homme. La relation à l'existence donnée est bouleversée, peut-être à cause de l'angoisse ou d'une détresse; dans tous les cas, c'est pour des motifs de défense qu'on prend ses distances. On sait, justement, que les formes et les figures peuvent avoir une multiplicité de significations. Peut-être l'enfant invente-t-il aussi son monde de jeu pour se protéger contre la dureté de l'existence. On a envie de faire apparaître, par enchantement, des créatures qui ne sont pas soumises à la mort, et de forger une réalité qui permette d'échapper à la répétition désespérée, signe de sclérose et de mort.

La conception positive et univoque du monde exclut la métamorphose; à supposer qu'une nouveauté appa-

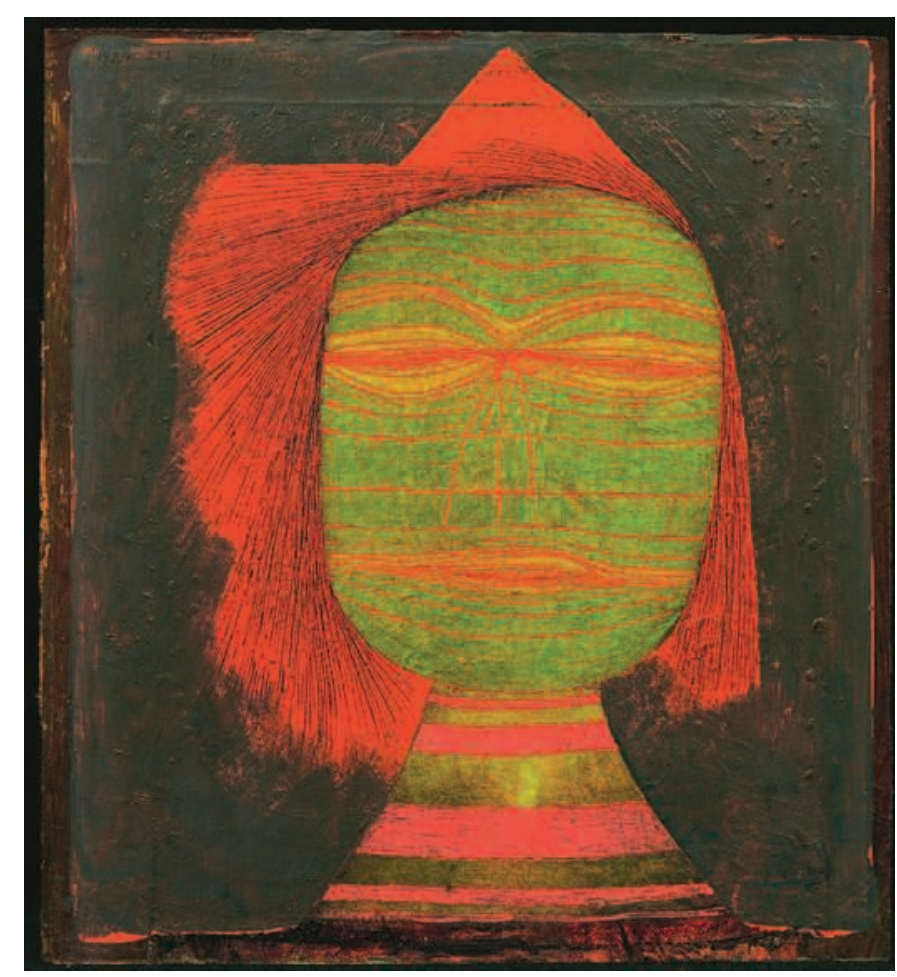

Fig. 16 Paul Klee, Le Masque de l'acteur, 1924, MoMA, The Sidney and Harriet Janis Collection, 1967, MoMA, New York / Scala, Florence.

raisse, elle est immédiatement écrasée et figée dans le cadre tout fait de la raison. Chez Klee, la figure est un tremplin vers la métamorphose. Toute figure n'est que passagère, elle n'est que le prélude à l'imprévu. On se protège contre la crainte et les métamorphoses trop rapides en ayant recours à des formes minérales de durée : c'est là une expression du désir d'une demeure. Une résistance se fait jour contre le processus ludique des métamorphoses.

En général, on est prisonnier de sa forme et on croit que celle-ci est une loi et une mesure de toute vie. Mais un sentiment peut s'éveiller, qui parcourt l'homme de temps à autre comme un éclair : le sentiment que tout être et toute forme en apparence assurée sont seulement contingents, et que le sens, pour l'homme, ne réside pas dans la conservation mais dans le changement métamorphique et la destruction des formes, un processus dont nous ne pouvons prévoir les phases. Par là, une autre attitude humaine s'instaure, on accepte le miracle imprévu comme une norme, alors que le devenir organique logique est ramené à un fait contingent, ou interprété comme une incapacité de l'homme à transformer son espèce. La sexualité, c'est-à-dire la production d'hommes, apparaît dès lors comme la 


\section{TEXTES DE CARL EINSTEIN}

désespérante monotonie de la conservation de l'espèce. Pour se défendre d'un tel désespoir, on forge des créatures fantastiques qui nous importent davantage, d'un point de vue psychique, que notre propre forme persistante, qui nous attache à un destin par trop étroit. Cela signifie que, dans la production de créatures nouvelles, l'homme manifeste le deuil désespéré que lui inspire sa corporéité figée et son dégoût de celle-ci. Les mêmes sentiments s'imposaient sans doute chez les premiers habitants de l'Asie Mineure et de la Grèce, encore barbares et anticlassiques, qui forgeaient des chimères et des monstres pour faire pendant à l'homme et à la norme classique. Ces monstres qui brillaient auparavant comme les héros des mythes furent plus tard rabaissés au rang de caricatures par l'homme enfermé dans les bornes classiques, qui les mit à l'écart ou les dissimula dans les royaumes souterrains de Pluton ou du diable. Avec l'essor de la conscience, l'imagination apparaît comme une fonction illogique et donc inférieure, ses créations sont rejetées comme des caprices insensés. Nous retournons alors au stade de la contreréaction, le règne du diable, qui avait été proscrit, et on retrouve là les forces décisives que le rationalisme avait à tort bannies et dévalorisées.

Dans la mythologie tardive, on a toujours davantage humanisé les forces de la nature et les esprits et on leur a imposé comme norme la figure humaine. Cette limitation a atteint son sommet avec l'art grec tardif et la Renaissance, ces vaines standardisations focalisées sur la beauté humaine. Tout comme on rejetait les forces et les créatures incomprises dans les sphères inférieures de la superstition, on se méfiait des dons qui permettaient de transgresser magiquement le monde imposé.

Dans les miniatures de Klee, nous assistons au retour des démons oniriques qui ressuscitent dans leurs forces anciennes et se chargent de la réalité la plus forte. Le réel ne signifie plus désormais une configuration logique univoque; il est recouvert et dominé par des visions issues de profondeurs oubliées. L'échelle de valeurs est renversée et l'irrationnel, en l'occurrence, la vision et la constitution du non encore connu, devient le fondement de l'existence.

Les primitifs, tournés vers le passé, aimaient conserver anxieusement dans une image les esprits des morts quand ils cessaient d'être visibles, afin de préserver la densité du sang; nous, au contraire, nous nous efforçons de dessiner les esprits que nous ne connaissons pas encore pour qu'ils prennent réalité.

Une telle attitude devient possible quand on ne fait plus confiance au savoir des dieux dont l'existence pré- détermine toutes les visions et les présuppose de façon nécessaire. Une telle liberté de donner forme et d'inventer n'est possible que lorsqu'on ose rejeter le dogmatisme divin et, du même coup, toute prédétermination de l'existence. Désormais, on se fie au flot de l'intuition imprévisible et, au lieu de reproduire avec respect, on transforme. On a dépassé le stade classicisant de Pygmalion, qui modèle la poupée de cire avec tant de ressemblance qu'elle se met à respirer, et on retourne à l'époque magique où l'on voyait surgir des créatures inattendues d'une branche ou d'une boule de glaise.

Nous avons parlé des formes de Klee, mais il nous reste encore à expliquer le clair et l'obscur de ces feuillets. La nuit est une obscurité émanant et irradiant des êtres comme une propriété. Puis nous rencontrons d'étranges figures qui poussent dans un désert pâle et atone; ou bien d'autres dont les facettes étincellent comme des soleils; ce sont des formes dans lesquelles sont emprisonnés des astres, ou peut-être l'étoile d'un ancêtre qui s'est incarné en eux. Les hommes peuvent pousser en tant que plantes ou connaître le repos des minéraux, briller en astres ou s'éteindre en lunes. Cela signifie que l'homme est un acrobate des états temporels : autant de rayons qui émanent de lui. Voilà pourquoi son destin est de se soumettre à tous les états et à toutes les métamorphoses; l'homme est le jeu rêvé d'une métamorphose incessante.

Chez Klee, nous observons un trait fondamental de l'existence contemporaine, la primitivisation, c'est-àdire le dégoût de la civilisation trop différenciée. On fuit la masse infinie de la conscience mécanisée, chosifiée, qui est presque devenue sans âme. Chez Klee, cette primitivisation ne signifie pas un ornement lisse ou une perte de la forme, mais un changement fondamental. Désormais, il ne s'agit plus des prémisses d'une destruction des choses, il s'agit bien plutôt d'une transformation de la structure psychique, à partir de laquelle des figures nouvelles se fraient un passage dans la réalité. L'art ne signifie plus seulement une expression de soi sans entrave, il peut être quelque chose de plus profond, à savoir le suicide et l'anéantissement de l'homme déjà existant au profit de formations nouvelles et possibles. Ainsi, l'art reconquiert des forces longtemps inemployées, celles de la prédiction et de l'interprétation des rêves, il redevient un moyen de transformer le réel et d'instaurer, à la place de l'ancienne qui se meurt, une nouvelle réalité plus proche de l'homme et en adéquation avec son psychisme tout entier et pas seulement avec la raison bornée. L'art retrouve la force d'être un outil magique et une prophétie de ce qui est à venir. 


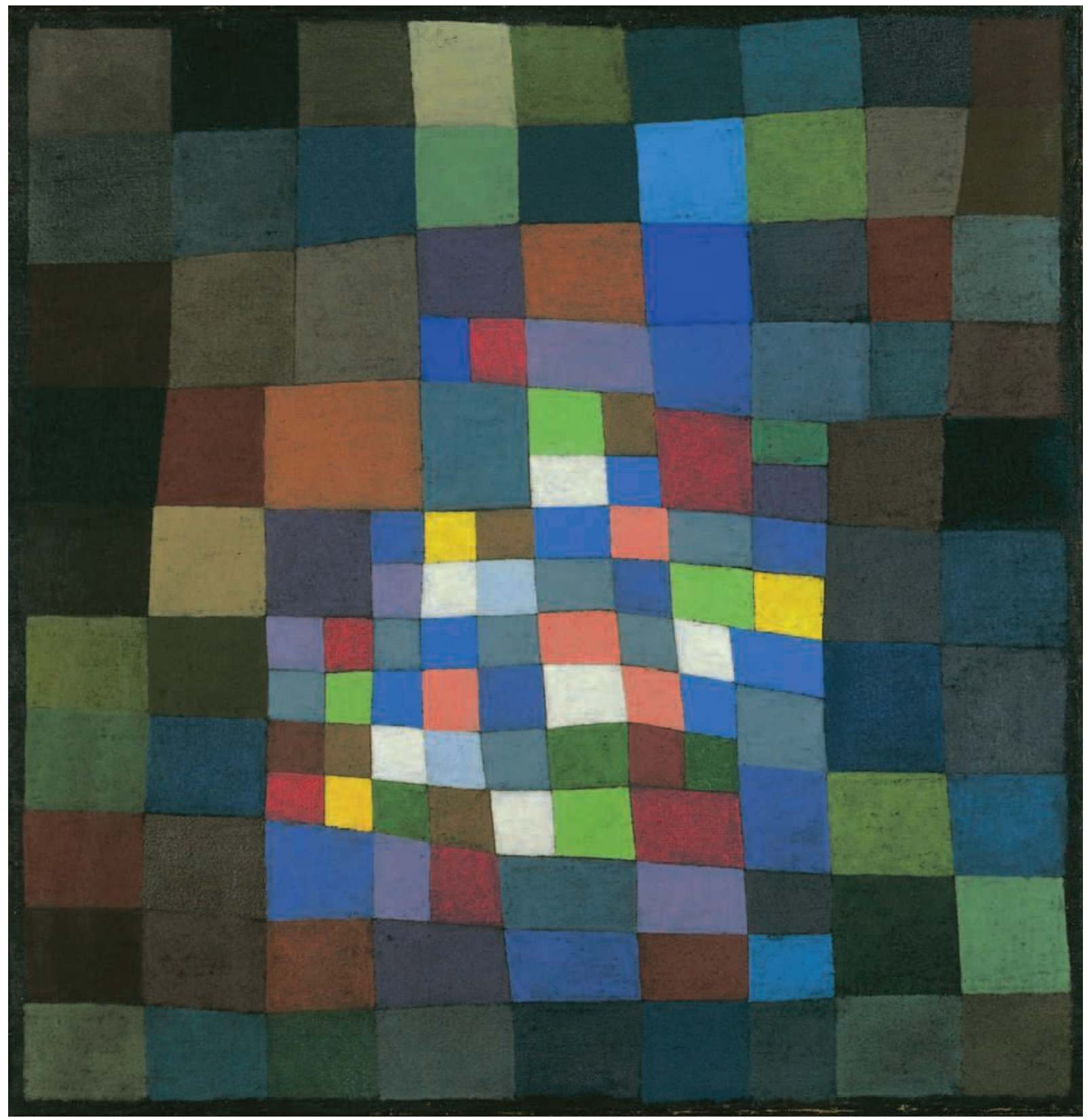

Fig. 17 Paul Klee, Floraison, 1934, Winterthur, Kunstmuseum Winterthur, don du Dr. Emil et de Clara Friedrich-Jezler, 1973. 